\title{
6-Hydroxydopamine Induces Neurodegeneration in Terminally Differentiated SH-SY5Y Neuroblastoma Cells via Enrichment of the Nucleosomal Degradation Pathway: a Global Proteomics Approach
}

\author{
Kasthuri Bai Magalingam ${ }^{1,2} \cdot$ Sushela Devi Somanath ${ }^{3} \cdot$ Premdass Ramdas $^{4} \cdot$ Nagaraja Haleagrahara $^{5}$. \\ Ammu Kutty Radhakrishnan ${ }^{2,6}$ (D)
}

Received: 25 April 2021 / Accepted: 22 December 2021 / Published online: 8 March 2022

(c) The Author(s) 2022

\begin{abstract}
The SH-SY5Y human neuroblastoma cells have been used for decades as a cell-based model of dopaminergic neurons to explore the underlying science of cellular and molecular mechanisms of neurodegeneration in Parkinson's disease (PD). However, data revealing the protein expression changes in 6-OHDA induced cytotoxicity in differentiated SH-SY5Y cells remain void. Therefore, we investigated the differentially regulated proteins expressed in terminally differentiated SH-SY5Y cells (differ-SH-SY5Y neural cells) exposed to 6-hydroxydopamine (6-OHDA) using the LC-MS/MS technology and construed the data using the online bioinformatics databases such as PANTHER, STRING, and KEGG. Our studies demonstrated that the neuronal development in differ-SH-SY5Y neural cells was indicated by the overexpression of proteins responsible for neurite formations such as calnexin (CANX) and calreticulin (CALR) besides significant downregulation of ribosomal proteins. The enrichment of the KEGG ribosome pathway was detected with significant downregulation $(p<0.05)$ of all the 21 ribosomal proteins in differ-SH-SY5Y neural cells compared with undifferentiated cells. Whereas in the PD model, the pathological changes induced by 6-OHDA were indicated by the presence of unfolded and misfolded proteins, which triggered the response of $10 \mathrm{kDa}$ heat shock proteins (HSP), namely HSPE1 and HSPA9. Moreover, the 6-OHDA-induced neurodegeneration in differ-SH-SY5Y neural cells also upregulated the voltage-dependent anion-selective channel protein 1 (VDAC1) protein and enriched the KEGG systemic lupus erythematosus (SLE) pathway that was regulated by 17 histone proteins $(p<0.05)$ in differ-SH-SY5Y neural cells. These results suggest that the nucleosomal degradation pathway may have regulated the 6-OHDA induced neurodegeneration in PD cell-based model, which is reflected by increased apoptosis and histone release in differ-SH-SY5Y neural cells.
\end{abstract}

Keywords Parkinson's disease (PD) · 6-Hydroxydopamine (6-OHDA) · Human neuroblastoma cells (SH-SY5Y)

\section{Introduction}

Over the last 20 years, a significant amount of data has emerged suggesting different cellular models that are valuable in evaluating potential drug targets in modulating

Ammu Kutty Radhakrishnan

ammu.radhakrishnan@monash.edu

1 School of Postgraduate Studies, International Medical University, Kuala Lumpur, Malaysia

2 Jeffrey Cheah School of Medicine and Health Sciences, Monash University, Malaysia, Bandar Sunway, Malaysia

3 Pathology Division, School of Medicine, International Medical University, Kuala Lumpur, Malaysia the disease course in PD. Undeniably, the immortalized SHSY5Y human neuroblastoma cell line has been an excellent choice for the preliminary drug screening for PD. The SHSY5Y cell line demonstrates a moderate activity of crucial neuronal markers that usually exists in dopaminergic cells

4 Division of Applied Biomedical Sciences and Biotechnology, School of Health Sciences, International Medical University, Kuala Lumpur, Malaysia

5 College of Public Health, Medicine and Veterinary Sciences, James Cook University, Townsville, QLD 4811, Australia

6 Monash-Industry Palm Oil Education and Research Platform (MIPO), Monash University Malaysia, Bandar Sunway, Selangor, Malaysia 
such as dopamine- $\beta$-hydroxylase (Katsuyama et al. 2021), tyrosine hydroxylase (Khwanraj et al. 2015), choline acetyl-transferase (Filograna et al. 2015) and noradrenaline (Kovalevich and Langford 2013). Whether undifferentiated or differentiated SH-SY5Y neuroblastoma should be used as a substitute to dopaminergic neurons in PD-related studies remain controversial. However, most neuroscience researchers have suggested that it is of great importance to utilize the differentiated SH-SY5Y human neuroblastoma cells that resemble human dopamine neurons for PD studies (Magalingam et al. 2020).

A fantastic wealth of information has been accumulated on different techniques and protocols to develop terminally matured dopamine neurons. Cheung et al. have pointed out that retinoic acid (RA) induced differentiation of SHSY5Y cells demonstrated extensive outgrowth of neurite and augmentation of neuronal markers such as neuronal nuclei, enolase, synaptophysin and synaptic-associated protein-97 (Cheung et al. 2009). Studies have also shown that RA-induced differentiation exhibits low proliferative rate and increased expression of genes related to synaptic vesicle cycle, dopamine synthesis/ degradation and dopamine transporter (DAT) (Lopes et al. 2017), as well as higher tyrosine hydroxylase, which is the critical enzyme in the synthesis of dopamine neurotransmitter (Khwanraj et al. 2015). Furthermore, our previous studies have delineated that the RA-induced differentiation on $\mathrm{SH}$ SY5Y cells in a low-serum culture medium demonstrated enhanced neurite projection with longer varicosities connecting the adjacent cells, reduced proliferation rate as well as increased levels of dopamine and alpha-synuclein. Moreover, the differentiated neural cells also expresses dopaminergic characteristics at the genetic level as evidenced in upregulation of the DRD2 fold change expression (Magalingam et al. 2020). Our findings correlate with numerous previous studies that suggested that differentiated SH-SY5Y cell line possesses the closest resemblance with dopaminergic cells and is suitable for neurodegenerative related studies (Khwanraj et al. 2015; Lopes et al. 2017).

Although there is ample evidence on various approaches to establishing differentiated neuronal cells, data on the 6-OHDA induced cytotoxicity on differentiated SH-SY5Y cells remains scarce. Lopes et al. (2017) and Cheung et al. (2009) have shown that 6-OHDA have varied susceptibility on RA-differentiated SH-SY5Y cells. Other studies have documented the biochemical changes induced by 6-OHDA on undifferentiated SH-SY5Y cells. These studies have shown that 6-OHDA-induced cytotoxicity is mediated by mitochondrial fragmentation (Gomez-Lazaro et al. 2008), autophagy (Arsikin et al. 2012), mitochondrial respiration (Iglesias-González et al. 2012), generation of free radicals (Storch et al. 2000) and changes in lipid classes
(Xicoy et al. 2020). Since there is no data available on the proteomic changes implicated by 6-OHDA on differentiated SH-SY5Y cells, our findings contribute to filling the knowledge gap on the molecular, biological and pathways regulated by the 6-OHDA on RA-differentiated SH-SY5Y neuroblastoma cells.

The proteomic study is an emerging field of large-scale protein expression study that provides an unprecedented insight into the cellular structural and functional framework. Intrinsically, proteins within the cell play a crucial role in providing cellular structure, movement and communication, and participate in metabolism, respiration, signal transduction and reproduction activities (Aslam et al. 2017). The differential expression of proteomes in cells is a signal of significant alterations in cellular activity. The label-free liquid chromatography coupled to tandem mass spectrometry (LC-MS/MS) technology quantified and revealed thousands of global proteins across samples from digested peptides and matched the detected peptide/protein identity using automated database searching (Sinitcyn et al. 2018). Subsequently, the Protein ANalysis THrough Evolutionary Relationships (PANTHER) (Mi et al. 2019) bioinformatic database was applied to analyse the differentially regulated proteins for their functional annotation using gene ontology (GO) (Pomaznoy et al. 2018). The Search Tool for the Retrieval of Interacting Genes/Proteins (STRING) (Szklarczyk et al. 2019) and Kyoto Encyclopaedia of Genes and Genomes (KEGG) (Kanehisa 2019) search engines were utilized to understand the protein-protein interaction, enriched bio-pathway and molecular function.

In the present study, we performed label-free global protein profiling of terminally differentiated SH-SY5Y (differSH-SY5Y) neural cells and the cell model of PD using 6-hydroxydopamine-induced neurodegeneration of differSH-SY5Y cells based on a shotgun proteomic methodology. The main objective of this study is to identify the novel biomarkers and pathways involved in the neuronal development of SH-SY5Y neuroblastoma cells and cellular model of PD. The functional role and enriched canonical pathway of the differentially expressed proteins identified from these studies were further explored using bioinformatic tools. This study further authenticates the use of the 6-OHDA induced cytotoxicity on differ-SH-SY5Y cell model as an important tool in the investigation of potential drug targets for PD.

\section{Methods}

\section{Cell Culture}

The SH-SY5Y neuroblastoma cells (Cat \# CRL-2266) were grown in a complete culture medium containing $88 \%$ of Dulbecco Modified Eagle Medium (DMEM) supplemented with 
$4.5 \mathrm{~g} / \mathrm{L}$ glucose and L-glutamine without sodium pyruvate (Corning, Corning, NY, U.S.A), 10\% heat-inactivated fetal bovine serum (FBS, Biosera, Nuaille, France), 1\% Penicillinstreptomycin (P/S) (Gibco, Carlsbad, USA) and 1\% nonessential amino acid (NEAA, GIBCO, Carlsbad, USA) $37{ }^{\circ} \mathrm{C}$ in a humidified $5 \% \mathrm{CO}_{2}$ incubator. The culture medium was replaced every 3 -days, and cells were sub-cultured once it reaches $70 \%$ confluence.

\section{Differentiation of SH-SY5Y Neuroblastoma Cells}

The establishment of differ-SH-SY5Y neural cells was performed by seeding $1 \times 10^{5}$ cells $/ \mathrm{mL}$ of SH-SY5Y human neuroblastoma cells in a complete culture medium in a T75 flask. After $24 \mathrm{~h}$ of cell seeding, the cells were exposed to a differentiation medium containing 95\% of DMEM, 3\% heat-activated FBS, $1 \% \mathrm{P} / \mathrm{S}, 1 \% \mathrm{NEAA}$ and $10 \mu \mathrm{M}$ of all trans-retinoic acid (RA, Sigma Aldrich, St. Louis, USA) for 6-days. After 6-days of the differentiation phase, the differ-SH-SY5Y neural cells express dopaminergic characteristics at morphological, biochemical and genetic levels (Magalingam et al. 2020).

\section{Preparation and Treatment Protocol of 6-Hydroxydopamine}

6-Hydroxydopamine (6-OHDA) (Sigma Aldrich, St. Louis, USA) was freshly prepared using chilled $0.15 \%$ ascorbic acid (Sigma Aldrich, St. Louis, USA) and sterilized by filtering through a syringe filter fitted with a $0.2-\mu \mathrm{M}$ filter and stored in the dark at $4{ }^{\circ} \mathrm{C}$ to protect it from light. On day 7 (after the 6-days of differentiation phase), the differ-SH-SY5Y cells were exposed to $10 \mu \mathrm{g} / \mathrm{mL}$ of 6-OHDA in a serum-free culture medium for $24 \mathrm{~h}$. The undifferentiated cells were maintained in a serum-free culture medium without 6-OHDA for the same duration of time. The concentration of 6-OHDA $(10 \mu \mathrm{g} / \mathrm{mL})$ was selected based on the preliminary studies that displayed inhibition of differ-SH-SY5Y neural cell proliferation by about $50 \%$.

\section{Protein Extraction}

On day 8 , the undifferentiated, differ-SH-SY5Y, and 6-OHDA exposed differ-SH-SY5Y neural cells were harvested from the $\mathrm{T} 75$ flasks and recovered by centrifugation (1000 $\mathrm{g}$ for $10 \mathrm{~min}$ at $4{ }^{\circ} \mathrm{C}$ ). The supernatant was discarded, and the total protein was extracted from the cells using the EasyPrep Mini MS Sample Prep kit (Thermo Fisher Scientific, USA). Briefly, $100 \mu \mathrm{L}$ of lysis buffer (provided with the kit) and $1 \mu \mathrm{L}$ of universal nuclease (provided with the kit) were added to the cell pellets and thoroughly mixed until the sample's viscosity reduced. The extracted protein samples were aliquoted and stored at $-80{ }^{\circ} \mathrm{C}$ until further use.

\section{Determination of Protein Concentration}

The extracted proteins' concentration was estimated using the Pierce BCA Protein Kit (Thermo Fisher Scientific, USA) (El-Rami et al. 2017). The absorbance was measured at $562 \mathrm{~nm}$ using a microplate reader (SpectramaxM, CA, USA). A standard curve was prepared by plotting the average corrected absorbance measurements of BSA standards vs concentration in $\mu \mathrm{g} / \mathrm{mL}$ to determine the "unknown samples' protein concentrations.

\section{Protein Reduction and Alkylation}

The extracted proteins were subjected to reduction and alkylation steps followed by protein digestion and a "cleanup" procedure using the EasyPrep Mini MS Sample Prep kit protocol. The samples were removed from the $-80^{\circ} \mathrm{C}$ freezer and thawed at room temperature. Once samples were liquefied, $100 \mu \mathrm{g}$ of protein from each sample was transferred into appropriately labelled sterile $1.5-\mathrm{mL}$ tubes, and the final volume for each sample was adjusted to $100 \mu \mathrm{L}$ with lysis solution (provided with the kit). Then, $50 \mu \mathrm{L}$ of reduction solution (provided with the kit) was added to each tube and mixed gently. Following this, $50 \mu \mathrm{L}$ of alkylation solution (provided with the kit) was added to the tubes, and the tubes were gently mixed. The tubes were incubated at $95{ }^{\circ} \mathrm{C}$ using a heat block for $10 \mathrm{~min}$ to reduce and alkylation reactions. Following this, the samples were removed from the heat block to cool to room temperature and subjected to Trypsin/ Lys-C protein digestion procedure.

\section{Trypsin/Lys-C Protein Digestion}

For the digestion step, $500 \mu \mathrm{L}$ of the enzyme reconstitution solution (provided with the kit) was added to a vial containing Trypsin/Lys-C-Protease mix (provided with the kit). Then, $50 \mu \mathrm{L}$ of the reconstituted Trypsin/Lys-C-Protease mix was added to each tube containing the samples, and the tubes were incubated at $37{ }^{\circ} \mathrm{C}$ with shaking for $3 \mathrm{~h}$ to allow protein digestion to take place. At the end of $3 \mathrm{~h}, 50 \mu \mathrm{L}$ of digestion stop solution (provided with the kit) was added to each tube to terminate the digestion process.

\section{Peptide Clean-up}

After the peptide digestion, any contaminants present in the samples were sequentially removed using the peptide clean-up column (provided with the kit). The peptide clean-up columns were labelled accordingly to avoid any sample mix-ups. As per the manufacturer recommended protocol, the white cap at the bottom of each peptide clean-up column was removed, and its green top cap 
loosened before each clean-up column was placed in individual $2 \mathrm{~mL}$ tubes. The tubes were centrifuged $(3000 \mathrm{~g}$ for $2 \mathrm{~min}$ ) to remove trapped liquid from the column, and the flow-through from each column was discarded. The digested peptides from each sample were transferred into the respective peptide clean-up column. The columns were centrifuged ( $1500 \mathrm{~g}$ for $2 \mathrm{~min})$, and the flow-through from each column was discarded. Next, wash solution A (provided with the kit) was added into each column before centrifugation, and the flow-through from each column was discarded. This step was repeated using wash solution B (provided with the kit). Before the elution step, the peptide columns were centrifuged to remove any residual fluid. Then, each column was placed on appropriately labelled sterile collection tubes, and elution solution (provided with the kit) was added to each column. The eluted peptide samples were collected by centrifugation, dried using a vacuum centrifuge and stored at $-80{ }^{\circ} \mathrm{C}$ before LC-MS/ MS analysis.

\section{Liquid Chromatography and Mass Spectrometry Analysis}

The digested peptides were loaded into an Agilent 1200 HPLC-Chip/MS Interface, coupled with Agilent 6550 iFunnel Q-TOF LC/MS (Agilent, Santa Clara, CA, USA). The column was equilibrated with $0.1 \%$ formic acid in water (solution A). The peptides were eluted from the column with $90 \%$ acetonitrile in $0.1 \%$ formic acid in water (solution B). Quadrupole-time of flight (Q-TOF) polarity was set at positive with capillary and fragmenter voltage being set at $1900 \mathrm{~V}$ and $360 \mathrm{~V}$, respectively, and $5 \mathrm{~L} / \mathrm{min}$ of gas flow with a temperature of $325^{\circ} \mathrm{C}$. The collision energy was determined at $3.7 \mathrm{~V} \mathrm{(100} \mathrm{Da),} \mathrm{and} \mathrm{reference} \mathrm{masses} \mathrm{with}$ positive polarity was set at 299.294457 and 1221.990637 . The peptide spectrum was analysed in auto MS mode ranging from $110-3000 \mathrm{~m} / \mathrm{z}$ for MS scan and $50-3000 \mathrm{~m} / \mathrm{z}$ for MS/MS scan.

\section{Data Computation}

The raw data of tryptic peptides were extracted and processed using PEAKS X software (Bioinformatics Solutions Inc., Waterloo, ON, Canada) using Uniprot, Swissprot and TrEMBL databases. The PEAKS X software allows for the determination of the protein abundance using the following search parameters: retention time lower bound: $\geq 0$, retention time upper bound: $\leq 55$, average area: $\geq 0$, charge lower bound: $\geq 1$, confident number samples per group: $\geq 1$, peptide identification count: $\geq 1$, protein significance: $\geq 20$, used peptides: $\geq 1$, fixed modification: Carbamidomethylation of cysteine residues and false discovery rate (FDR): $1 \%$ in three biological replicate injections. Protein abundance was computed using normalized spectral protein intensity (LFQ intensity). The obtained peptide/protein list was exported to Microsoft Excel to quantitate three biological replicates from the same samples were grouped in the same matrix. The protein data were filtered for at least two valid values, and protein only presented in one biological replicate was eliminated. The biological replicates from all samples were clustered under the same matrix, and the missing data were assigned with a random number derived from a normal distribution. The reason for the missing data measurement is due to low protein abundance in LC-MS/MS analysis.

\section{Biocomputational Protein analysis}

\section{Protein Functional Classification}

The identified proteins with $p<0.05$ against their respective controls were classified based on (i) molecular function, (ii) biological process and (iii) cellular component using Gene Ontology (GO) term analysis. The online bioinformatics tool PANTHER database (http://pantherdb.org) version 16 was used to elucidate the functions of these differentially expressed proteins in the differ-SH-SY5Y neural cells and 6-OHDA exposed differ-SH-SY5Y cells. Only the top 10 enriched GO terms were listed for each functional classification. All results displayed expressed adjusted $p$-value $<0.05$ as determined by Fischer's Exact test and FDR.

\section{Protein-Protein Interaction (PPI) Analysis}

The STRING database (STRINGv11.0) (https://string-db. org) was used to construct the PPI network in neuronal maturation and oxidative stress in 6-OHDA treated neural cells based on its physical binding and regulatory interaction. The Uniprot IDs of the differentially regulated proteins were inputted in the STRING database under the multiple protein analysis categories and followed by the selection of Homo Sapiens from organism pull-down selection. The basic settings that were used in the analysis of PPI are Network type: Full network, active interaction sources: text mining, neighbourhood, experiments, databases, co-expression, gene-fusion, co-occurrence, Minimum required interaction score: highest confidence (0.9) and K-mean clustering was specified as three clusters. Thick edges between the protein nodes demonstrate strong protein interaction. This protein 
cluster was uploaded into Cytoscape 3.8.0 to visualise the complex networks by integrating $\log 2$ fold-change data.

\section{Pathway Enrichment Analysis}

The pathway enrichment analysis of the differentially expressed proteins was performed using DAVID (Database for Annotation, Visualization and Integrated Discovery, https://david.ncifcrf.gov/) bioinformatic online database. The cluster with the most enriched proteins exhibiting strong PPI identified from STRING analysis was uploaded as an official gene symbol in DAVID resources. The enriched pathway curated by KEGG (Kyoto Encyclopaedia of Genes and Genomes, (https://www.kegg.jp/kegg/mapper/color. $\mathrm{html}$ ) was used to elucidate the differentially regulated protein molecular mechanisms.

\section{Statistical Analysis}

Statistical analysis comparing the quantitative data from differential protein expression between differ-SH-SY5Y neural cells vs undifferentiated SH-SY5Y cells and 6-OHDA treated differ-SH-SY5Y neural cells vs untreated differ-SH-SY5Y cells were performed using a two-tailed Student's $t$-test. All statistical analyses were performed with GraphPad Prism version 9.0. Differentially expressed proteins that displayed the difference in Log2 Fold change ( $<$ or $>0$ ) with $p<0.05$ were regarded as statistically significant.

\section{Results}

\section{Label-Free Spectrometry Identification and Quantification of Differentially Regulated Proteins}

The protein profiling of differ-SH-SY5Y neural cells vs undifferentiated SH-SY5Y cells and 6-OHDA induced neurodegeneration vs untreated differ-SH-SY5Y were analysed using PEAKS X + software. The label-free tandem liquid mass spectrometry (LC-MS/MS) identified a total of 3261 and 3873 proteins for the differentiated and undifferentiated SH-SY5Y neural cells, respectively. Around 687 and 941 proteins were common in all the biological triplicates in differ-SH-SY5Y and undifferentiated SHSY5Y cells, respectively. When we compared the standard protein sets between differentiated and undifferentiated SH-SY5Y neural cells, we found a total of 189 (Table 1) common proteins appeared in both protein data sets with
86 statistically significantly differentiated proteins with $p<0.05$. Among 86 significantly regulated proteins, 63 proteins displayed a significant upregulation, while 23 proteins were downregulated. The $\log _{2}$ fold-change distribution of differentially regulated protein revealed seven proteins exhibiting a difference of onefold change [RS12 (40S ribosomal protein S12), RL10 (60S ribosomal protein L10), RL12 (60S ribosomal protein L12), DX39B (Spliceosome RNA helicase DDX39B), RSSA (40S ribosomal protein SA), CALX (Calnexin) and CALR (Calreticulin)], 21 proteins with the 0.5 - onefold change, 60 proteins showing 0.5 - onefold-change and 101 proteins with no statistical difference fold change (Fig. 1A).

On the other hand, the 6-OHDA treated differ-SH-SY5Y neural cells yielded 3636 proteins with 293 proteins shared the common data sets. When the protein profiles from the 6-OHDA treatment were compared with that of the untreated differentiated SH-SY5Y cells, a total of 188 proteins matched both protein data sets with a total of 101 proteins (Table 2) exhibited statistical significance difference. Among 101 significantly regulated proteins, 63 proteins were upregulated, and 38 proteins downregulated. The Log2 fold-change distribution of differentially regulated proteins displays three proteins with the difference of onefold change [VDAC1 (Voltage-dependent anion-selective channel protein 1), HSPA9 (Stress-70 protein mitochondrial), HSPE1 (10 $\mathrm{kDa}$ heat shock protein mitochondrial)], 15 proteins with a 0.5 - onefold-change difference, 84 proteins showing 0.5 - onefold-change and 86 proteins with no statistical difference fold-change (Fig. 1B).

\section{Biocomputational Analysis of Differentially Regulated Proteins}

\section{Functional Annotation Analysis of Differentially Regulated Proteins Using PANTHER}

To further elucidate the changes in cellular processes, the gene ontology (GO) analysis of the 86 differentially expressed proteins $(p<0.05)$ in the differ-SH-SY5Y neural cells were investigated using the PANTHER bioinformatics database. These proteins were analysed based on changes in the GO term classification clusters' biological process, molecular function, and cellular component. The GOmolecular function of differ-SH-SY5Y neural cells showed a strong correlation to the structural modulation, activity and binding of ribosomes and ribosomal proteins. As for the GO-biological process, the differentially expressed proteins modulated the ribosomal assembly, biogenesis, 
Table 1 Up- and downregulated proteins in differ-SH-SY5Y neural cells with average log2 fold change protein ratios between replicates

\begin{tabular}{|c|c|c|c|c|c|}
\hline \multicolumn{6}{|c|}{ Upregulated proteins } \\
\hline Uniprot Accession & Description of protein & Symbol & Average mass & $P$ values & $\begin{array}{l}\log 2 \text { (Fold } \\
\text { change) }\end{array}$ \\
\hline P27824 & Calnexin & CANX & 67,568 & $4.5 \mathrm{E}-03$ & 1.571 \\
\hline P27797 & Calreticulin & CALR & 48,142 & 3.7E-02 & 1.072 \\
\hline P09455 & Retinol-binding protein 1 & RBP1 & 15,850 & $1.2 \mathrm{E}-02$ & 0.867 \\
\hline P07602 & Prosaposin & PSAP & 58,113 & $3.8 \mathrm{E}-03$ & 0.783 \\
\hline P30101 & Protein disulfide-isomerase A3 & PDIA3 & 56,782 & $3.3 \mathrm{E}-02$ & 0.539 \\
\hline P30044 & Peroxiredoxin-5 mitochondrial & PRDX5 & 22,086 & $2.8 \mathrm{E}-04$ & 0.483 \\
\hline P07237 & Protein disulfide-isomerase & $\mathrm{P} 4 \mathrm{HB}$ & 57,116 & $1.4 \mathrm{E}-02$ & 0.370 \\
\hline Q71DI3 & Histone H3.2 & HIST2H3A & 15,388 & $1.4 \mathrm{E}-02$ & 0.333 \\
\hline Q12905 & Interleukin enhancer-binding factor 2 & ILF2 & 43,062 & $2.0 \mathrm{E}-02$ & 0.330 \\
\hline P08670 & Vimentin & VIM & 53,652 & $2.3 \mathrm{E}-03$ & 0.282 \\
\hline Q15084 & Protein disulfide-isomerase A6 & PDIA6 & 48,121 & $5.8 \mathrm{E}-03$ & 0.209 \\
\hline Q06830 & Peroxiredoxin-1 & PRDX1 & 22,110 & $9.7 \mathrm{E}-04$ & 0.197 \\
\hline P14625 & Endoplasmin & HSP90B1 & 92,469 & $6.8 \mathrm{E}-03$ & 0.167 \\
\hline Q99714 & 3-hydroxyacyl-CoA dehydrogenase type-2 & HSD17B10 & 26,923 & $3.4 \mathrm{E}-03$ & 0.156 \\
\hline P09429 & High mobility group protein $\mathrm{B} 1$ & HMGB1 & 24,894 & $2.5 \mathrm{E}-02$ & 0.099 \\
\hline P23284 & Peptidyl-prolyl cis-trans isomerase B & PPIB & 23,743 & 7.0E-03 & 0.092 \\
\hline P61978 & Heterogeneous nuclear ribonucleoprotein $\mathrm{K}$ & HNRNPK & 50,976 & $3.9 \mathrm{E}-04$ & 0.085 \\
\hline P07910 & Heterogeneous nuclear ribonucleoproteins $\mathrm{C} 1 / \mathrm{C} 2$ & HNRNPC & 33,670 & 3.7E-02 & 0.072 \\
\hline P0DP23 & Calmodulin-1 & CALM1 & 16,838 & $2.6 \mathrm{E}-02$ & 0.066 \\
\hline P0DP24 & Calmodulin-2 & CALM2 & 16,838 & $2.6 \mathrm{E}-02$ & 0.066 \\
\hline P0DP25 & Calmodulin-3 & CALM3 & 16,838 & $2.6 \mathrm{E}-02$ & 0.066 \\
\hline Q16555 & Dihydropyrimidinase-related protein 2 & DPYSL2 & 62,294 & $1.9 \mathrm{E}-04$ & 0.045 \\
\hline P31946 & 14-3-3 protein beta/alpha & YWHAB & 28,082 & $5.8 \mathrm{E}-03$ & 0.031 \\
\hline \multicolumn{6}{|c|}{ Downregulated proteins } \\
\hline Uniprot Accession & Description of protein & Symbol & Average mass & $P$ values & $\begin{array}{l}\text { Log2 (Fold } \\
\text { change) }\end{array}$ \\
\hline P25398 & 40S ribosomal protein $\mathrm{S} 12$ & RPS12 & 14,515 & 2.7E-04 & -1.648 \\
\hline P30050 & 60S ribosomal protein L12 & RPL12 & 17,819 & 4.4E-03 & -1.290 \\
\hline Q13838 & Spliceosome RNA helicase DDX39B & DDX39B & 48,991 & $1.6 \mathrm{E}-02$ & -1.218 \\
\hline P27635 & $60 \mathrm{~S}$ ribosomal protein L10 & RPL10 & 24,604 & $3.6 \mathrm{E}-03$ & -1.047 \\
\hline P08865 & 40S ribosomal protein SA & RPSA & 32,854 & 2.3E-02 & -1.023 \\
\hline Q86VP6 & Cullin-associated NEDD8-dissociated protein 1 & CAND1 & 136,375 & $8.3 \mathrm{E}-05$ & -0.980 \\
\hline P46776 & $60 S$ ribosomal protein $\mathrm{L} 27 \mathrm{a}$ & RPL27A & 16,561 & 2.9E-05 & -0.889 \\
\hline P49321 & Nuclear autoantigenic sperm protein & NASP & 85,238 & $2.9 \mathrm{E}-02$ & -0.860 \\
\hline P05388 & $60 \mathrm{~S}$ acidic ribosomal protein $\mathrm{P} 0$ & RPLP0 & 34,274 & $3.2 \mathrm{E}-02$ & -0.837 \\
\hline P46782 & 40S ribosomal protein S5 & RPS5 & 22,876 & $3.6 \mathrm{E}-02$ & -0.816 \\
\hline P27695 & DNA-(apurinic or apyrimidinic site) lyase & APEX1 & 35,555 & 4.4E-02 & -0.769 \\
\hline P62913 & 60S ribosomal protein L11 & RPL11 & 20,252 & $1.1 \mathrm{E}-02$ & -0.735 \\
\hline Q9UJZ1 & Stomatin-like protein 2 mitochondrial & STOML2 & 38,534 & 7.1E-04 & -0.701 \\
\hline P05387 & $60 \mathrm{~S}$ acidic ribosomal protein $\mathrm{P} 2$ & RPLP2 & 11,665 & $9.0 \mathrm{E}-04$ & -0.690 \\
\hline P60842 & Eukaryotic initiation factor 4A-I & EIF4A1 & 46,154 & $2.1 \mathrm{E}-02$ & -0.662 \\
\hline P61088 & Ubiquitin-conjugating enzyme $\mathrm{E} 2 \mathrm{~N}$ & UBE2N & 17,138 & $2.8 \mathrm{E}-05$ & -0.628 \\
\hline Q16658 & Fascin & FSCN1 & 54,530 & $2.4 \mathrm{E}-02$ & -0.606 \\
\hline P30153 & $\begin{array}{l}\text { Serine/threonine-protein phosphatase 2A } 65 \\
\text { kDa regulatory subunit A alpha isoform }\end{array}$ & PPP2R1A & 65,309 & $4.8 \mathrm{E}-02$ & -0.591 \\
\hline P62081 & 40S ribosomal protein $\mathrm{S} 7$ & RPS7 & 22,127 & $1.1 \mathrm{E}-02$ & -0.580 \\
\hline P37108 & Signal recognition particle $14 \mathrm{kDa}$ protein & SRP14 & 14,570 & $1.3 \mathrm{E}-03$ & -0.578 \\
\hline
\end{tabular}


Table 1 (continued)

\begin{tabular}{|c|c|c|c|c|c|}
\hline \multicolumn{6}{|c|}{ Downregulated proteins } \\
\hline Uniprot Accession & Description of protein & Symbol & Average mass & $P$ values & $\begin{array}{l}\log 2 \text { (Fold } \\
\text { change) }\end{array}$ \\
\hline P62701 & 40S ribosomal protein $\mathrm{S} 4 \mathrm{X}$ isoform & RPS4X & 29,598 & $1.8 \mathrm{E}-04$ & -0.552 \\
\hline P62906 & $60 \mathrm{~S}$ ribosomal protein $\mathrm{L} 10 \mathrm{a}$ & RPL10A & 24,831 & $3.0 \mathrm{E}-03$ & -0.494 \\
\hline P49327 & Fatty acid synthase & FASN & 273,424 & $7.8 \mathrm{E}-03$ & -0.494 \\
\hline Q15366 & Poly(rC)-binding protein 2 & PCBP2 & 38,580 & $2.8 \mathrm{E}-03$ & -0.490 \\
\hline P38159 & RNA-binding motif protein X chromosome & RBMX & 42,332 & $1.2 \mathrm{E}-02$ & -0.484 \\
\hline P83731 & $60 \mathrm{~S}$ ribosomal protein L24 & RPL24 & 17,779 & $1.2 \mathrm{E}-02$ & -0.423 \\
\hline P35637 & RNA-binding protein FUS & FUS & 53,426 & $3.8 \mathrm{E}-02$ & -0.398 \\
\hline P61981 & $14-3-3$ protein gamma & YWHAG & 28,303 & 4.2E-02 & -0.394 \\
\hline P24752 & Acetyl-CoA acetyltransferase mitochondrial & ACAT1 & 45,200 & $1.9 \mathrm{E}-02$ & -0.392 \\
\hline P13639 & Elongation factor 2 & EEF2 & 95,338 & 4.8E-02 & -0.370 \\
\hline P52565 & Rho GDP-dissociation inhibitor 1 & ARHGDIA & 23,207 & $3.4 \mathrm{E}-03$ & -0.360 \\
\hline P15121 & Aldose reductase & AKR1B1 & 35,853 & $1.4 \mathrm{E}-05$ & -0.340 \\
\hline P14174 & Macrophage migration inhibitory factor & MIF & 12,476 & $2.3 \mathrm{E}-03$ & -0.340 \\
\hline Q99525 & Histone H4-like protein type $\mathrm{G}$ & HIST1H4G & 11,009 & 7.1E-03 & -0.329 \\
\hline P19338 & Nucleolin & NCL & 76,615 & $1.3 \mathrm{E}-03$ & -0.317 \\
\hline P05386 & $60 \mathrm{~S}$ acidic ribosomal protein $\mathrm{P} 1$ & RPLP1 & 11,514 & $2.3 \mathrm{E}-03$ & -0.315 \\
\hline Q14103 & Heterogeneous nuclear ribonucleoprotein D0 & HNRNPD & 38,434 & $3.3 \mathrm{E}-03$ & -0.306 \\
\hline P36578 & $60 \mathrm{~S}$ ribosomal protein $\mathrm{L} 4$ & RPL4 & 47,697 & $8.9 \mathrm{E}-03$ & -0.288 \\
\hline P62851 & 40S ribosomal protein $S 25$ & RPS25 & 13,742 & $1.2 \mathrm{E}-04$ & -0.286 \\
\hline P23396 & $40 \mathrm{~S}$ ribosomal protein $\mathrm{S} 3$ & RPS3 & 26,688 & 7.3E-03 & -0.279 \\
\hline P24534 & Elongation factor 1-beta & EEF1B2 & 24,764 & $9.9 \mathrm{E}-05$ & -0.274 \\
\hline P07195 & L-lactate dehydrogenase B chain & LDHB & 36,639 & 7.4E-03 & -0.269 \\
\hline P14618 & Pyruvate kinase PKM & PKM & 57,937 & $5.3 \mathrm{E}-03$ & -0.267 \\
\hline P00558 & Phosphoglycerate kinase 1 & PGK1 & 44,615 & $1.2 \mathrm{E}-02$ & -0.257 \\
\hline P09211 & Glutathione S-transferase P & GSTP1 & 23,356 & $9.3 \mathrm{E}-04$ & -0.255 \\
\hline Q01105 & Protein SET & SET & 33,489 & $3.3 \mathrm{E}-02$ & -0.243 \\
\hline P46781 & $40 \mathrm{~S}$ ribosomal protein $\mathrm{S} 9$ & RPS9 & 22,591 & $3.1 \mathrm{E}-02$ & -0.240 \\
\hline P0C0S5 & Histone H2A.Z & $\mathrm{H} 2 \mathrm{AFZ}$ & 13,553 & $5.8 \mathrm{E}-03$ & -0.234 \\
\hline P18124 & $60 \mathrm{~S}$ ribosomal protein $\mathrm{L} 7$ & RPL7 & 29,226 & $2.1 \mathrm{E}-02$ & -0.226 \\
\hline P50395 & Rab GDP dissociation inhibitor beta & GDI2 & 50,663 & $3.4 \mathrm{E}-03$ & -0.226 \\
\hline Q02878 & $60 \mathrm{~S}$ ribosomal protein $\mathrm{L} 6$ & RPL6 & 32,728 & $2.8 \mathrm{E}-02$ & -0.224 \\
\hline P06748 & Nucleophosmin & NPM1 & 32,575 & $2.1 \mathrm{E}-02$ & -0.196 \\
\hline P40227 & T-complex protein 1 subunit zeta & ССТ6А & 58,024 & $1.2 \mathrm{E}-02$ & -0.193 \\
\hline P30041 & Peroxiredoxin- 6 & PRDX6 & 25,035 & 4.3E-02 & -0.180 \\
\hline P62258 & 14-3-3 protein epsilon & YWHAE & 29,174 & $4.5 \mathrm{E}-02$ & -0.163 \\
\hline Q71UI9 & Histone H2A.V & $\mathrm{H} 2 \mathrm{AFV}$ & 13,509 & 4.5E-02 & -0.152 \\
\hline P11142 & Heat shock cognate $71 \mathrm{kDa}$ protein & HSPA8 & 70,898 & 8.7E-04 & -0.146 \\
\hline P40926 & Malate dehydrogenase mitochondrial & MDH2 & 35,503 & $1.8 \mathrm{E}-02$ & -0.138 \\
\hline P52272 & Heterogeneous nuclear ribonucleoprotein M & HNRNPM & 77,516 & 4.1E-02 & -0.105 \\
\hline P62805 & Histone H4 & HIST1H4A & 11,367 & $6.8 \mathrm{E}-03$ & -0.092 \\
\hline P10809 & $60 \mathrm{kDa}$ heat shock protein mitochondrial & HSPD1 & 61,055 & 4.3E-02 & -0.063 \\
\hline P06733 & Alpha-enolase & ENO1 & 47,169 & $1.2 \mathrm{E}-02$ & -0.045 \\
\hline P62277 & 40S ribosomal protein $\mathrm{S} 13$ & RPS13 & 17,222 & $1.4 \mathrm{E}-02$ & -0.041 \\
\hline
\end{tabular}




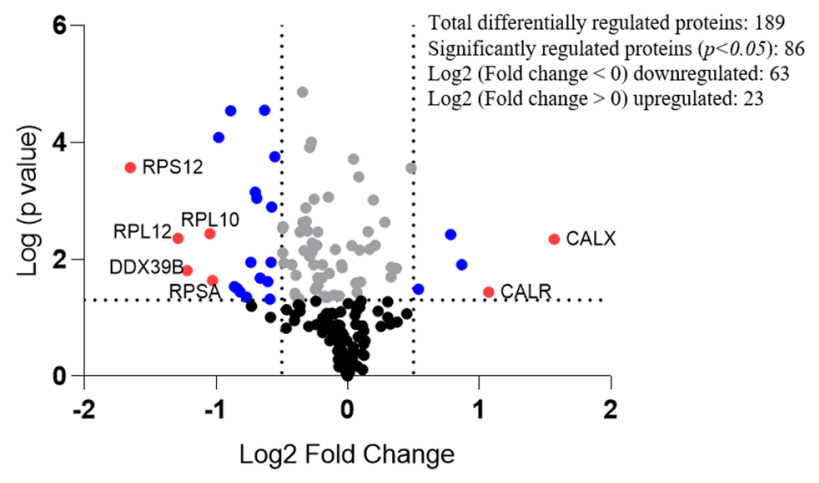

(A)

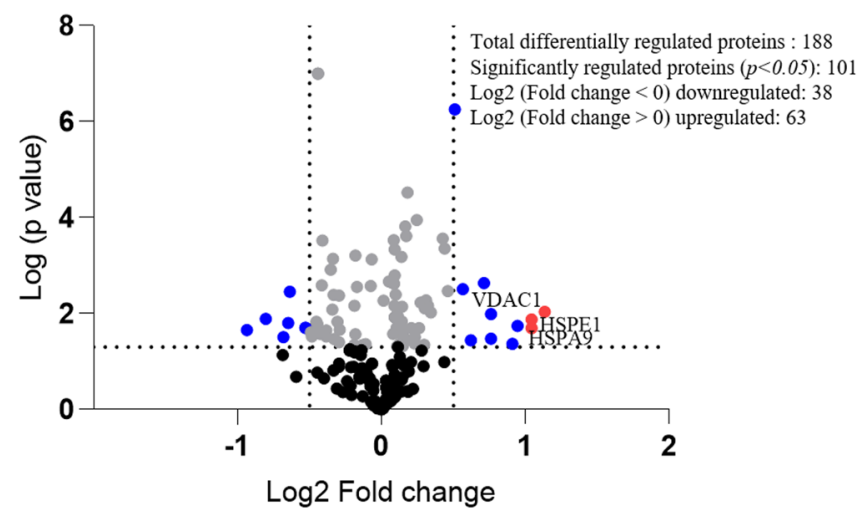

(B)
Fig. 1 Quantitative proteomic analysis of neuronal development and 6-OHDA induced neurodegeneration on differ-SH-SY5Y neural cells. Volcano plot shows the differentially regulated protein in (A) DifferSH-SY5Y neural cells (B) 6-OHDA induced neurodegeneration in differ-SH-SY5Y neural cells. The horizontal coordinate (x-axis) represents the difference in fold change (logarithmic transformation at the base of 2), and the vertical coordinate (Y-axis) is the significant

glycolytic process (ATP generation) and DNA synthesis (purine nucleoside and ribonucleotide diphosphate phosphorylation and metabolic process). These molecular and biological processes were identified as concentrated at cytosolic ribosome units, as shown in the GO-cellular component (Fig. 2A).

Next, for the 6-OHDA induced neurodegeneration in differ-SH-SY5Y, the GO-molecular function showed the association of the differentially regulated proteins to the DNA structural alterations (structural molecule, purine, nucleoside, nucleotide), changes in structural constituents of ribosomes, metabolic processes (carbohydrate derivative binding), calcium cell signalling pathway (Calcium ion binding), metal ion homeostasis (metal ion binding) and unfolded protein binding. For the GO-biological process, the analysis revealed 6-OHDA induced neurodegeneration causes enrichment of the cellular mechanisms that identify and respond to misfolded/unfolded proteins (i.e. protein folding, cellular response to topologically incorrect protein, cellular response to unfolded proteins, endoplasmic reticulum unfolded protein response), metabolic activity (glycolytic activity, ATP generation), DNA structural changes (purine ribonucleoside diphosphate metabolic process), and apoptotic nucleosome assembly. The proteins localized in GO-cellular components were identified to be mitochondria (i.e. proton-transporting ATP synthase complex, mitochondrial prohibitin complex, mitochondria cristae), neuronal synapse or ribbon synapse (dense body), ribosome (i.e. eukaryotic translation elongation factor 1 complex, cytosolic RNA splicing (pIC1n-Sn protein complex, U7 snRNP) and endoplasmic reticulum (Fig. 2B). difference of $p$-value (logarithmic transformation at the base of 10). Proteins that are onefold significantly regulated are presented as red dots, while those that had 0.5 to onefold change are shown as blue dots. Proteins with fold change less than 0.5-fold change are presented as grey dots. Black dots represent proteins that do not have a statistically significant fold-change

\section{STRING Protein-Protein Interaction (PPI) Network Analysis}

The STRING PPI network in differ-SH-SY5Y neural cells generated a total of 396 edges and 86 nodes with a PPI enrichment $p$-value of $<1.00 \times 10^{-16}$, which was generated using the highest confidence (0.9) category (Fig. 3A). The PPI enrichment clusters showed a local clustering coefficient of 0.658. A total of three clusters were generated using K-means clustering. Proteins in cluster I include peroxiredoxin-6 (PRDX6), peroxiredoxin-5 mitochondrial (PRDX5), rho GDP-dissociation inhibitor 1 (ARHGDIA), peroxiredoxin-1 (PRDX1), 14-3-3 protein epsilon (YWHAE), 14-3-3 protein beta/alpha (YWHAB), 14-3-3 protein gamma (YWHAG), Rab GDP dissociation inhibitor beta (GDI2) and Glutathione S-transferase P (GSTP1), whilst proteins in cluster II comprises of EEF1B2 (Elongation factor 1-beta), EEF2 (Elongation factor 2), EIF4A1 (Eukaryotic initiation factor 4A-I), PPP2RIA (Serine/ threonine-protein phosphatase $2 \mathrm{~A} 65 \mathrm{kDa}$ regulatory subunit A alpha isoform), RPL10 (60S ribosomal protein L10), RPL10A (60S ribosomal protein L10a), RPL11 (60S ribosomal protein L11), RPL12 (60S ribosomal protein L12), RPL24 (Ribosomal protein L24), RPL27A (Ribosomal protein L27a), RPL4 (L ribosomal proteins), RPL6 (60S ribosomal protein L6), RPL7 (60S ribosomal protein L7), RPLP0 (60S acidic ribosomal protein P0), RPLP1 (60S acidic ribosomal protein P1), RPLP2 (60S acidic ribosomal protein P2), RPS12 (Ribosomal protein S12), RPS13 (Ribosomal protein S13), RPS25 (Ribosomal protein S25), RPS3 (40S ribosomal protein S3), RPS4X 
Table 2 Up- and downregulated proteins in 6-OHDA induced neurodegeneration on differ-SH-SY5Y cells with average 6-OHDA/untreated protein ratios between replicates

\begin{tabular}{|c|c|c|c|c|c|}
\hline \multicolumn{6}{|c|}{ Upregulated proteins } \\
\hline $\begin{array}{l}\text { Uniprot } \\
\text { Accession }\end{array}$ & Description of protein & Symbol & $\begin{array}{l}\text { Average } \\
\text { mass }\end{array}$ & $P$ value & $\begin{array}{l}\log 2 \text { (Fold } \\
\text { change) }\end{array}$ \\
\hline P21796 & Voltage-dependent anion-selective channel protein 1 & VDAC1 & 61546 & $9.20 \mathrm{E}-03$ & 1.135 \\
\hline P38646 & Stress- 70 protein mitochondrial & HSPA9 & 147362 & $2.00 \mathrm{E}-02$ & 1.044 \\
\hline P61604 & $10 \mathrm{kDa}$ heat shock protein mitochondrial & HSPE1 & 21864 & $1.33 \mathrm{E}-02$ & 1.044 \\
\hline P30049 & ATP synthase subunit delta mitochondrial & ATP5F1D & 34980 & 1.80E-02 & 0.945 \\
\hline P08758 & Annexin A5 & ANXA5 & 71874 & 4.29E-02 & 0.911 \\
\hline P49411 & Elongation factor Tu mitochondrial & TUFM & 99084 & 3.32E-02 & 0.762 \\
\hline P40939 & Trifunctional enzyme subunit alpha mitochondrial & HADHA & 166000 & $1.03 \mathrm{E}-02$ & 0.761 \\
\hline Q9Y277 & Voltage-dependent anion-selective channel protein 3 & VDAC3 & 61318 & $2.31 \mathrm{E}-03$ & 0.712 \\
\hline P35232 & Prohibitin & PHB & 59608 & $3.61 \mathrm{E}-02$ & 0.622 \\
\hline Q99623 & Prohibitin-2 & PHB2 & 66592 & $3.10 \mathrm{E}-03$ & 0.565 \\
\hline Q9UJZ1 & Stomatin-like protein 2 mitochondrial & STOML2 & 77068 & $5.50 \mathrm{E}-07$ & 0.511 \\
\hline P24752 & Acetyl-CoA acetyltransferase mitochondrial & ACAT1 & 90400 & $3.38 \mathrm{E}-03$ & 0.462 \\
\hline $\mathrm{O} 15240$ & Neurosecretory protein VGF & VGF & 134516 & $4.43 \mathrm{E}-04$ & 0.439 \\
\hline P05141 & ADP/ATP translocase 2 & SLC25A5 & 65704 & $2.74 \mathrm{E}-04$ & 0.425 \\
\hline O00571 & ATP-dependent RNA helicase DDX3X & DDX3X & 146488 & $9.43 \mathrm{E}-03$ & 0.343 \\
\hline P04792 & Heat shock protein beta- 1 & HSPB 1 & 45566 & $6.85 \mathrm{E}-03$ & 0.324 \\
\hline P06576 & ATP synthase subunit beta mitochondrial & ATP5F1B & 113120 & $5.29 \mathrm{E}-03$ & 0.310 \\
\hline P25705 & ATP synthase subunit alpha mitochondrial & ATP5F1A & 119502 & 7.78E-03 & 0.302 \\
\hline P49327 & Fatty acid synthase & FASN & 546848 & 4.45E-02 & 0.296 \\
\hline P07355 & Annexin A2 & ANXA2 & 77208 & $5.93 \mathrm{E}-03$ & 0.273 \\
\hline P11021 & Endoplasmic reticulum chaperone $\mathrm{BiP}$ & HSPA5 & 144666 & $2.02 \mathrm{E}-02$ & 0.261 \\
\hline P30101 & Protein disulfide-isomerase A3 & PDIA3 & 113564 & $1.13 \mathrm{E}-04$ & 0.246 \\
\hline P45880 & Voltage-dependent anion-selective channel protein 2 & VDAC2 & 63134 & $3.08 \mathrm{E}-02$ & 0.233 \\
\hline Q08211 & ATP-dependent RNA helicase A & DHX9 & 281916 & 4.21E-02 & 0.230 \\
\hline P27824 & Calnexin & CANX & 135136 & $2.00 \mathrm{E}-02$ & 0.209 \\
\hline Q9BVA1 & Tubulin beta- $2 \mathrm{~B}$ chain & TUBB2B & 99906 & $3.02 \mathrm{E}-05$ & 0.180 \\
\hline Q15084 & Protein disulfide-isomerase A6 & PDIA6 & 96242 & $3.45 \mathrm{E}-02$ & 0.171 \\
\hline P16104 & Histone $\mathrm{H} 2 \mathrm{AX}$ OS=Homo sapiens & $\mathrm{H} 2 \mathrm{AFX}$ & 30290 & $2.43 \mathrm{E}-04$ & 0.171 \\
\hline P14625 & Endoplasmin & HSP90B1 & 184938 & $1.53 \mathrm{E}-04$ & 0.166 \\
\hline Q13509 & Tubulin beta- 3 chain & TUBB3 & 100866 & $3.50 \mathrm{E}-02$ & 0.158 \\
\hline P23246 & Splicing factor proline- and glutamine-rich & SFPQ & 152300 & $2.12 \mathrm{E}-02$ & 0.158 \\
\hline P62805 & Histone $\mathrm{H} 4$ & HIST1H4A & 22734 & 4.75E-02 & 0.148 \\
\hline Q9P0M6 & Core histone macro-H2A.2 & $\mathrm{H} 2 \mathrm{AFY} 2$ & 80116 & $7.24 \mathrm{E}-03$ & 0.143 \\
\hline Q9BUF & Tubulin beta- 6 chain & TUBB6 & 99714 & $1.45 \mathrm{E}-02$ & 0.141 \\
\hline 5 P07602 & Prosaposin & PSAP & 116226 & $6.53 \mathrm{E}-04$ & 0.138 \\
\hline Q99714 & 3-hydroxyacyl-CoA dehydrogenase type-2 & HSD17B10 & 53846 & $1.81 \mathrm{E}-02$ & 0.128 \\
\hline P23284 & Peptidyl-prolyl cis-trans isomerase B & PPIB & 47486 & 4.24E-02 & 0.128 \\
\hline O75367 & Core histone macro-H2A.1 & H2AFY & 79234 & $2.54 \mathrm{E}-02$ & 0.127 \\
\hline P10809 & $60 \mathrm{kDa}$ heat shock protein mitochondrial & HSPD1 & 122110 & $1.10 \mathrm{E}-02$ & 0.112 \\
\hline P68104 & Elongation factor 1-alpha 1 & EEF1A1 & 100282 & 4.78E-02 & 0.111 \\
\hline
\end{tabular}


Table 2 (continued)

\begin{tabular}{|c|c|c|c|c|c|}
\hline \multicolumn{6}{|c|}{ Upregulated proteins } \\
\hline $\begin{array}{l}\text { Uniprot } \\
\text { Accession }\end{array}$ & Description of protein & Symbol & $\begin{array}{l}\text { Average } \\
\text { mass }\end{array}$ & $P$ value & $\begin{array}{l}\text { Log2 (Fold } \\
\text { change) }\end{array}$ \\
\hline P06899 & Histone H2B type 1-J & HIST1H2BJ & 27808 & $1.13 \mathrm{E}-02$ & 0.108 \\
\hline P23527 & Histone $\mathrm{H} 2 \mathrm{~B}$ type $1-\mathrm{O}$ & HIST1H2BO & 27812 & $1.13 \mathrm{E}-02$ & 0.108 \\
\hline P33778 & Histone $\mathrm{H} 2 \mathrm{~B}$ type 1-B & HIST1H2BB & 27900 & $1.13 \mathrm{E}-02$ & 0.108 \\
\hline Q16778 & Histone $\mathrm{H} 2 \mathrm{~B}$ type $2-\mathrm{E}$ & HIST2H2BE & 27,840 & $1.13 \mathrm{E}-02$ & 0.108 \\
\hline P62829 & $60 \mathrm{~S}$ ribosomal protein L23 & RPL23 & 29,730 & $1.98 \mathrm{E}-02$ & 0.106 \\
\hline P27797 & Calreticulin & CALR & 96,284 & $4.01 \mathrm{E}-03$ & 0.099 \\
\hline O60814 & Histone $\mathrm{H} 2 \mathrm{~B}$ type $1-\mathrm{K}$ & HIST1H2BK & 27,780 & 1.19E-02 & 0.094 \\
\hline P57053 & Histone $\mathrm{H} 2 \mathrm{~B}$ type F-S & H2BFS & 27,888 & 1.19E-02 & 0.094 \\
\hline P58876 & Histone $\mathrm{H} 2 \mathrm{~B}$ type 1-D & HIST1H2BD & 27,872 & 1.19E-02 & 0.094 \\
\hline P62807 & Histone $\mathrm{H} 2 \mathrm{~B}$ type $1-\mathrm{C} / \mathrm{E} / \mathrm{F} / \mathrm{G} / \mathrm{I}$ & HIST1H2BC & 27,812 & 1.19E-02 & 0.094 \\
\hline Q5QNW6 & Histone $\mathrm{H} 2 \mathrm{~B}$ type $2-\mathrm{F}$ & HIST2H2BF & 27,840 & 1.19E-02 & 0.094 \\
\hline Q93079 & Histone $\mathrm{H} 2 \mathrm{~B}$ type $1-\mathrm{H}$ & HIST1H2BH & 27,784 & 1.19E-02 & 0.094 \\
\hline Q99877 & Histone $\mathrm{H} 2 \mathrm{~B}$ type $1-\mathrm{N}$ & HIST1H2BN & 27,844 & 1.19E-02 & 0.094 \\
\hline Q99879 & Histone H2B type 1-M & HIST1H2BM & 27,978 & 1.19E-02 & 0.094 \\
\hline P60709 & Actin cytoplasmic 1 & ACTB & 83,474 & $4.58 \mathrm{E}-04$ & 0.092 \\
\hline P63261 & Actin cytoplasmic 2 & ACTG1 & 83,586 & $1.59 \mathrm{E}-03$ & 0.092 \\
\hline Q71U36 & Tubulin alpha-1A chain & TUBA1A & 100,272 & $6.86 \mathrm{E}-03$ & 0.091 \\
\hline P68032 & Actin alpha cardiac muscle 1 & ACTC1 & 84,038 & 2.39E-03 & 0.087 \\
\hline P68363 & Tubulin alpha-1B chain & TUBA1B & 100,304 & 2.94E-04 & 0.085 \\
\hline P07437 & Tubulin beta chain & TUBB & 99,342 & $2.66 \mathrm{E}-02$ & 0.081 \\
\hline Q05639 & Elongation factor 1-alpha 2 & EEF1A2 & 100,940 & $2.25 \mathrm{E}-03$ & 0.069 \\
\hline Q00610 & Clathrin heavy chain 1 & CLTC & 383,226 & $2.15 \mathrm{E}-03$ & 0.052 \\
\hline P62249 & 40S ribosomal protein $\mathrm{S} 16$ & RPS16 & 32,890 & 5.39E-03 & 0.013 \\
\hline \multicolumn{6}{|c|}{ Downregulated proteins } \\
\hline Uniprot Accession & Description of protein & Symbol & Average mass & $P$ value & Log2 (Fold Change) \\
\hline P32119 & Peroxiredoxin-2 & PRDX2 & 43,784 & 2.19E-02 & -0.935 \\
\hline P55072 & Transitional endoplasmic reticulum ATPase & $\mathrm{VCP}$ & 178,644 & $1.29 \mathrm{E}-02$ & -0.803 \\
\hline P30041 & Peroxiredoxin- 6 & PRDX6 & 50,070 & 3.09E-02 & -0.683 \\
\hline P19338 & Nucleolin & NCL & 153,230 & $1.56 \mathrm{E}-02$ & -0.648 \\
\hline P36578 & $60 \mathrm{~S}$ ribosomal protein $\mathrm{L} 4$ & RPL4 & 95,394 & $3.50 \mathrm{E}-03$ & -0.637 \\
\hline P31150 & Rab GDP dissociation inhibitor alpha & GDI1 & 101,166 & $1.97 \mathrm{E}-02$ & -0.527 \\
\hline P60028 & Rab GDP dissociation inhibitor alpha & GDI1 & 101,166 & 1.97E-02 & -0.527 \\
\hline P62753 & $40 \mathrm{~S}$ ribosomal protein $\mathrm{S} 6$ & RPS6 & 57,362 & $2.45 \mathrm{E}-02$ & -0.490 \\
\hline P14174 & Macrophage migration inhibitory factor & MIF & 24,952 & 3.02E-02 & -0.482 \\
\hline P62826 & GTP-binding nuclear protein Ran & RAN & 48,846 & $1.48 \mathrm{E}-02$ & -0.454 \\
\hline P60174 & Triosephosphate isomerase & TPI1 & 61,582 & $2.52 \mathrm{E}-02$ & -0.450 \\
\hline P00338 & L-lactate dehydrogenase A chain & LDHA & 73,378 & $1.66 \mathrm{E}-02$ & -0.446 \\
\hline P0DP23 & Calmodulin-1 & CALM1 & 33,676 & 9.96E-08 & -0.442 \\
\hline P0DP24 & Calmodulin-2 & CALM2 & 33,676 & $9.96 \mathrm{E}-08$ & -0.442 \\
\hline P0DP25 & Calmodulin-3 & CALM3 & 33,676 & $9.96 \mathrm{E}-08$ & -0.442 \\
\hline Q16555 & Dihydropyrimidinase-related protein 2 & DPYSL2 & 124,588 & $2.66 \mathrm{E}-02$ & -0.427 \\
\hline
\end{tabular}


Table 2 (continued)

\begin{tabular}{|c|c|c|c|c|c|}
\hline \multicolumn{6}{|c|}{ Downregulated proteins } \\
\hline Uniprot Accession & Description of protein & Symbol & Average mass & $P$ value & Log2 (Fold Change) \\
\hline P62841 & 40S ribosomal protein $\mathrm{S} 15$ & RPS15 & 34,080 & $2.58 \mathrm{E}-03$ & -0.413 \\
\hline Q06830 & Peroxiredoxin-1 & PRDX1 & 44,220 & 3.01E-04 & -0.411 \\
\hline P07195 & L-lactate dehydrogenase B chain & LDHB & 73,278 & $2.98 \mathrm{E}-02$ & -0.383 \\
\hline P62304 & Small nuclear ribonucleoprotein $\mathrm{E}$ & SNRPE & 21,608 & $2.24 \mathrm{E}-02$ & -0.383 \\
\hline Q13263 & Transcription intermediary factor 1 -beta & TRIM28 & 177,100 & $1.22 \mathrm{E}-03$ & -0.351 \\
\hline Q12905 & Interleukin enhancer-binding factor 2 & ILF2 & 86,124 & $8.24 \mathrm{E}-03$ & -0.342 \\
\hline P06733 & Alpha-enolase & ENO1 & 94,338 & 7.24E-04 & -0.336 \\
\hline P46782 & 40S ribosomal protein S5 & RPS5 & 45,752 & 4.07E-03 & -0.331 \\
\hline P29373 & Cellular retinoic acid-binding protein 2 & CRABP2 & 31,386 & $3.38 \mathrm{E}-02$ & -0.327 \\
\hline P12277 & Creatine kinase B-type & $\mathrm{CKB}$ & 85,288 & $1.49 \mathrm{E}-02$ & -0.306 \\
\hline P30153 & $\begin{array}{l}\text { Serine/threonine-protein phosphatase } 2 \mathrm{~A} 65 \mathrm{kDa} \\
\text { regulatory subunit A alpha isoform }\end{array}$ & PPP2R1A & 130,618 & $3.98 \mathrm{E}-02$ & -0.295 \\
\hline P52565 & Rho GDP-dissociation inhibitor 1 & ARHGDIA & 46,414 & $4.18 \mathrm{E}-03$ & -0.293 \\
\hline P06744 & Glucose-6-phosphate isomerase & GPI & 126,294 & $2.18 \mathrm{E}-02$ & -0.290 \\
\hline P63104 & 14-3-3 protein zeta/delta & YWHAZ & 55,490 & $4.51 \mathrm{E}-02$ & -0.189 \\
\hline Q14103 & Heterogeneous nuclear ribonucleoprotein D0 & HNRNPD & 76,868 & $6.85 \mathrm{E}-03$ & -0.188 \\
\hline P62851 & 40S ribosomal protein $\mathrm{S} 25$ & RPS & 27,484 & $6.19 \mathrm{E}-04$ & -0.180 \\
\hline P68431 & Histone H3.1 & HIST1H3A & 30,808 & $2.69 \mathrm{E}-02$ & -0.180 \\
\hline P37802 & Transgelin-2 & TAGLN & 44,782 & 2.79E-03 & -0.170 \\
\hline P62081 & $40 \mathrm{~S}$ ribosomal protein $\mathrm{S} 7$ & RPS & 44,254 & $4.50 \mathrm{E}-02$ & -0.133 \\
\hline P61978 & Heterogeneous nuclear ribonucleoprotein $\mathrm{K}$ & HNRNPK & 101,952 & $4.36 \mathrm{E}-02$ & -0.111 \\
\hline P62318 & Small nuclear ribonucleoprotein Sm D3 & SNRPD & 27,832 & $2.65 \mathrm{E}-03$ & -0.074 \\
\hline P13639 & Elongation factor 2 & $\mathrm{EEF}$ & 190,676 & $7.45 \mathrm{E}-04$ & -0.068 \\
\hline
\end{tabular}

(Ribosomal protein S4), RPS5 (Ribosomal protein S5), RPS7 (40S ribosomal protein S7), RPS9 (Ribosomal protein S9), RPSA (40S ribosomal protein SA) and SRP14 (Signal recognition particle $14 \mathrm{kDa}$ protein). Cluster III contains proteins such as DDX39B (Spliceosome RNA helicase DDX39B), FUS (RNA-binding protein FUS), HNRNPC (Heterogeneous nuclear ribonucleoproteins C1/ C2), HNRNPD (Heterogeneous nuclear ribonucleoprotein D0), HNRNPK (Heterogeneous nuclear ribonucleoprotein K), HNRNPM (Heterogeneous nuclear ribonucleoprotein M), HSPA8 (Heat shock cognate $71 \mathrm{kDa}$ protein), ILF2 (Interleukin enhancer-binding factor 2), RBMX (RNAbinding motif protein) and PCBP2 (Poly(rC)-binding protein 2) (Fig. 3A). As cluster II exhibits the highest confidence edges with strong populated PPI, proteins in this cluster were further analysed for the pathway enrichment using the DAVID TOOL and KEGG databases.

The STRING PPI networks of the 6-OHDA induced neurodegeneration in differ-SH-SY5Y neural cells that generated a total of 245 edges and 97 nodes with PPI enrichment $p$-value $<1.0 \times 10^{-16}$ is shown in Fig. $3 \mathrm{~B}$. The networks were derived using the highest confidence (0.9) category that demonstrates three distinct clusters generated using K-means clustering. The PPI enrichment clusters exhibited a local clustering coefficient of 0.676 that generated three distinct clusters using K-means clustering. Proteins in cluster I include CALR (calreticulin), CANX (calnexin), HSP90B1 (endoplasmin), HSPA5 (78 kDa glucose-regulated protein), HSPA9 (stress-70 protein, mitochondrial), HSPD1 (60 kDa heat shock protein), HSPE1 (10 kDa heat shock protein), PDIA3 (protein disulfide-isomerase A3), PDIA6 (protein disulfide-isomerase A6), PPIB (peptidyl-prolyl cis-trans isomerase B), SLC25A5 (ADP/ATP translocase 2), VDAC1 (voltage-dependent anion-selective channel protein 1), VDAC2 (voltage-dependent anion-selective channel protein 2), VDAC3 (voltage-dependent anion-selective channel protein 3), ATP5A1 (ATP synthase subunit alpha, mitochondrial), ATP5B (ATP synthase subunit beta, mitochondrial) ATP5D (ATP synthase subunit delta, mitochondrial) and VGF (neurosecretory protein VGF). Cluster II includes RPL4 (L ribosomal proteins), RPS16 (Ribosomal protein S16), RPS6 (40S ribosomal protein S6), RPS15 (Ribosomal protein S15), RPS25 (40S ribosomal protein S25), RPS16 (40S ribosomal protein S16), RPL23 (60S ribosomal protein L23), EEF1A1 (Elongation factor 1-alpha 1), EEF2 (Elongation factor 2), RPS7 (40S ribosomal protein S7), EEF1A2 (Elongation factor 1-alpha 2), whilst proteins in cluster III include histone proteins such as H2AFX (Histone 
H2AX), H2AFY (Core histone macro-H2A.1), H2AFY2 (Core histone macro-H2A.2), H2BFS (Histone H2B type F-S), HIST1H2BB (Histone H2B type 1-B), HIST1H2BC (Histone H2B type 1-C), HIST1H2BD (Histone H2B type 1-D), HIST1H2BH (Histone H2B type 1-H), HIST1H2BJ (Histone H2B type 1-J), HIST1H2BK (Histone H2B type 1-K), HIST1H2BM (Histone H2B type 1-M), HIST1H2BN (Histone H2B type 1-N), HIST1H2BO (Histone H2B type 1-O), HIST1H3A (Histone cluster $1 \mathrm{H} 3$ family member a), HIST1H4A (Histone cluster $1 \mathrm{H} 4$ family member a), HIST2H2BE (Histone H2B type 2-E), HIST2H2BF (Histone H2B type 2-F), RAN (GTP-binding nuclear protein Ran) and TRIM28 (Transcription intermediary factor 1-beta). The strong PPI edges and highest number of protein recruitment were noted in cluster III compared to other clusters; hence proteins populated in this cluster were subjected to pathway enrichment analysis.

\section{Discussion}

The SH-SY5Y human neuroblastoma cell line is regarded as versatile neuronal cells that can be applied in multitude neuroscience research. However, it is imperative to differentiate

(A)

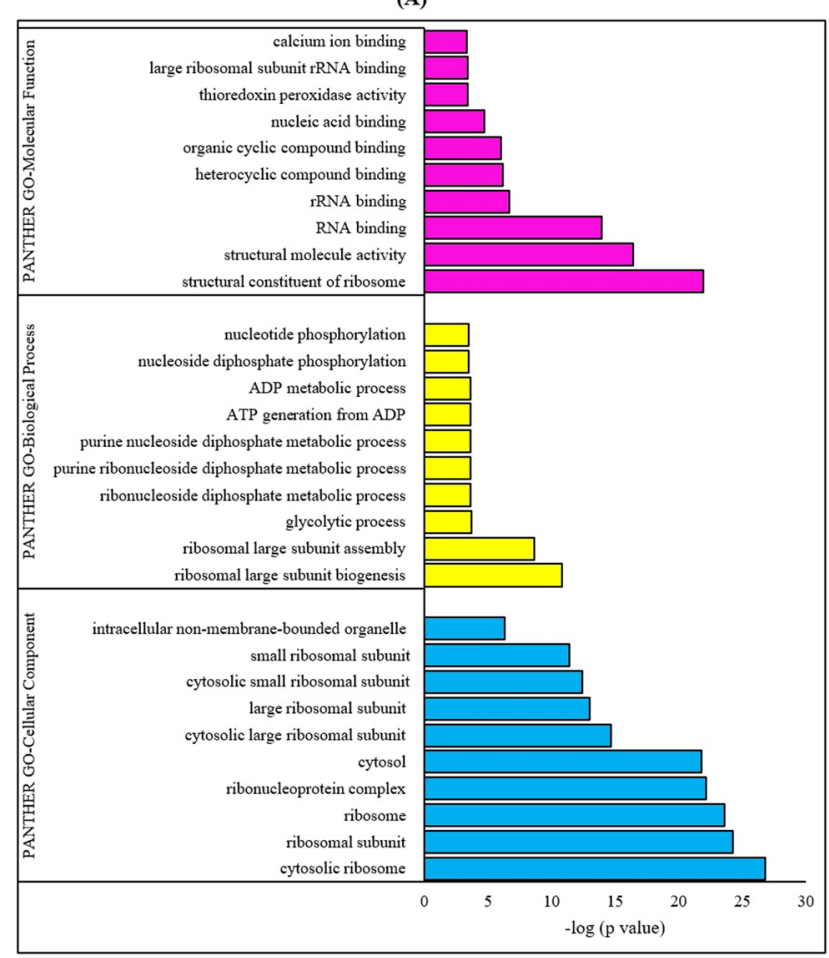

Fig. 2 The GO classification terms of molecular function, biological process, and cellular component using PANTHER online database on differentially regulated proteins $(p<0.05)$ in (A) Differ-SH-
Fig. 3 (A) The STRING PPI analysis of differentially regulated proteins in differ-SH-SY5Y neural cells vs undifferentiated SH-SY5Y cells. (B) STRING Protein-protein interaction network of 6-OHDA induced neurodegeneration on differ-SH-SY5Y neural cells. The STRING PPI analysis of differentially regulated proteins in 6-OHDA treatment on differ-SH-SY5Y neural cells vs untreated differ-SHSY5Y cells. The red dotted circles show protein clusters with the highest confidence (0.9) interactions. The edges were drawn with different line thickness that indicates the strength of data support

the highly proliferative neuroblastoma cells that entails a number matured neuron characteristics such as extension of neurite projections, increased electrical potential, expression of neuron-specific markers and neurotransmitters (Kovalevich and Langford 2013). In this regard, several studies have highlighted that RA enhances the expression of dopaminergic (Lopes et al. 2017), adrenergic and cholinergic characteristics (Hashemi et al. 2003; HES et al. 2018) in human SH-SY5Y neuroblastoma cells. On that note, our research team has previously reported that the SH-SY5Y neuroblastoma cells differentiated in RA containing low serum medium for 6 days significantly enhanced the dopamine and $\alpha$-synuclein levels besides upregulating the dopamine receptor D2 (DRD2) gene expression (Magalingam et al. 2020). In continuation of our previous study, this study is aimed to gain further insights into the differential

(B)

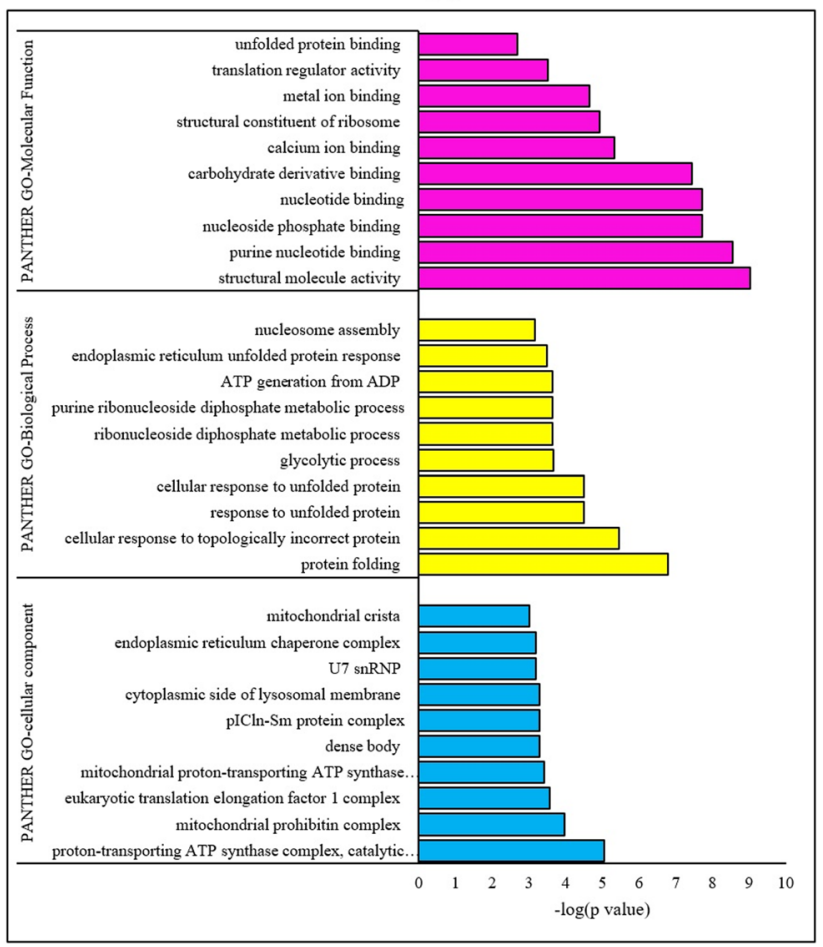

SY5Y neural cells in comparison with undifferentiated SH-SY5Y neural cells and (B) 6-OHDA treated differ-SH-SY5Y neural cells in comparison with untreated differ-SH-SY5Y neural cells 


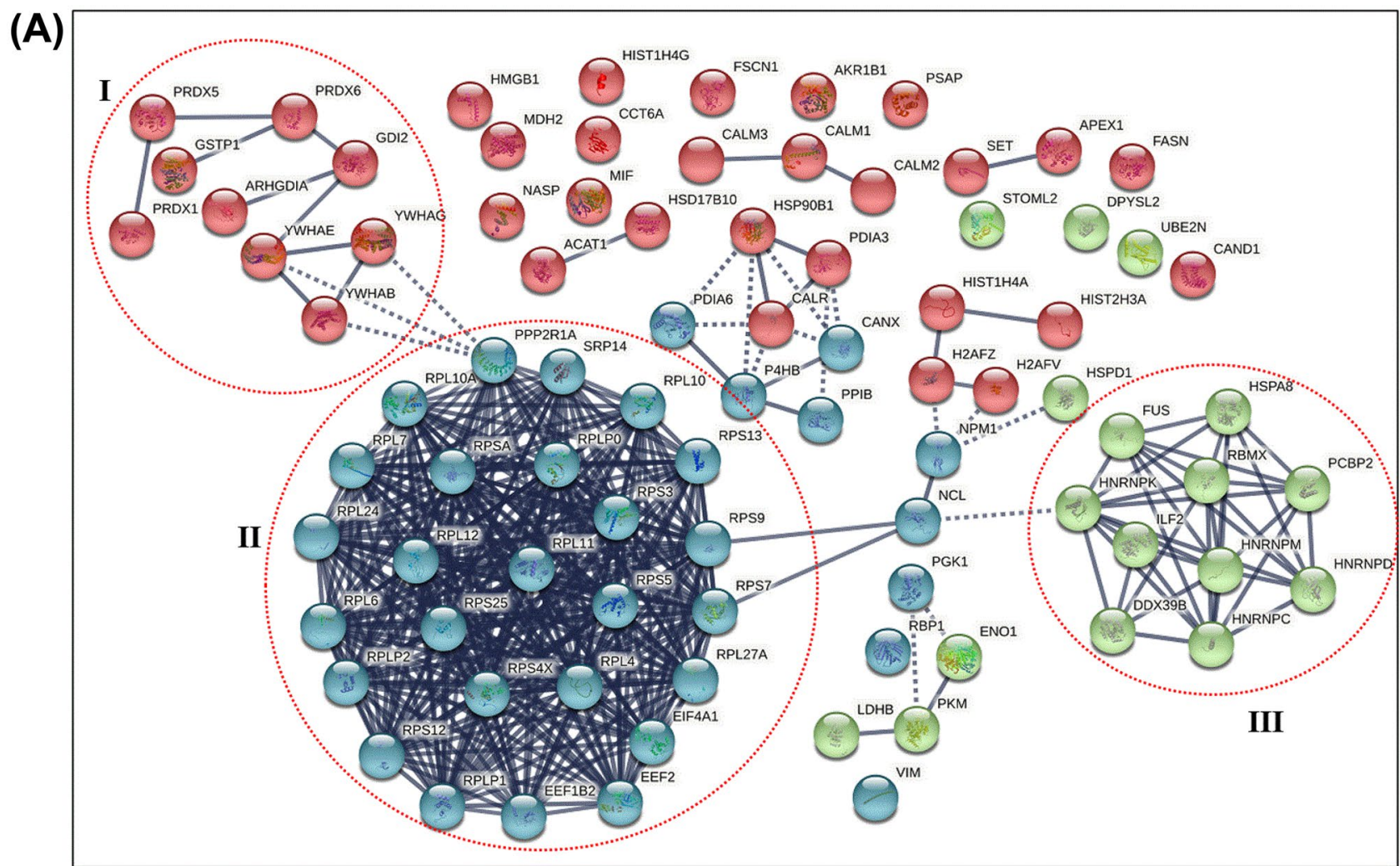

(B)

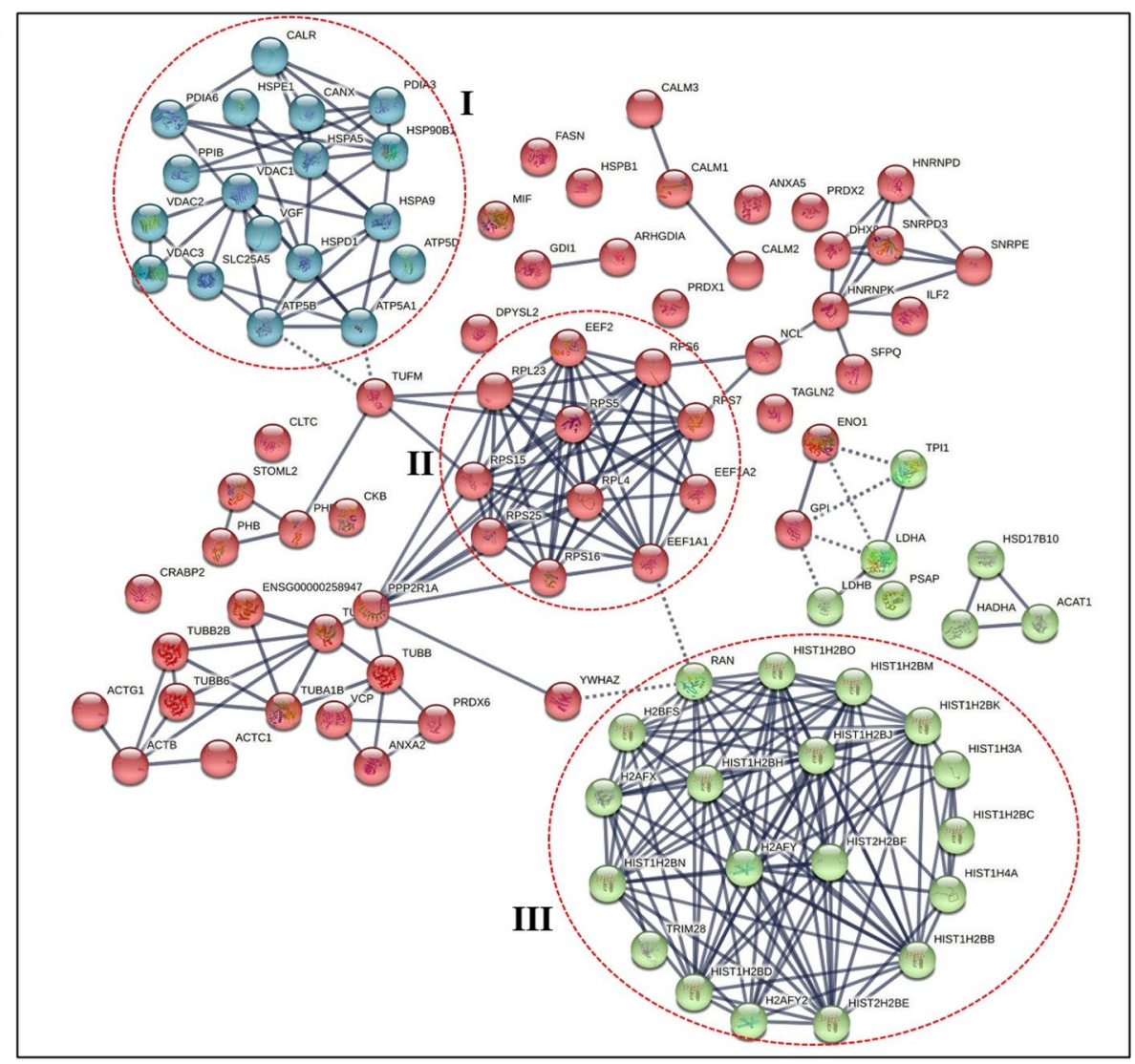


proteome expression pattern implicated by RA in human neuroblastoma cells. Next, we explored the biological and molecular pathway induced by 6-OHDA in differ-SH-SY5Y, that is known as cellular model of PD.

Our findings suggest that the RA induced differentiation of SH-SY5Y cells exhibited a significant increase in the expression of two crucial proteins, calnexin and calreticulin proteins (Fig. 1A). Calreticulin, an essential $\mathrm{Ca}^{2+}$ binding chaperone in the endoplasmic reticulum, is a vital entity of the calreticulin/calnexin cycle (Xiao et al. 2017). Nevertheless, the calreticulin/calnexin cycle plays a vital role in folding newly synthesized proteins for cellular differentiation or organ development (Dudek and Michalak 2013). Emerging studies have shown that calreticulin mediated suppression of oncogenic N-MYC (MYCN) resulted in increased neurite length and differentiation marker GAP-43 (Lee et al. 2019). A study on the calreticulin knock-out mouse model displayed embryonic lethality with remarkable defects in the heart, brain and body wall, suggesting the pivotal role of calreticulin in the development of the nervous system (Rauch et al. 2000). On the other hand, calnexin's role has been associated with nerve cells' myelination, as being deficient in the calnexin gene leads to myelinopathy (Kraus et al. 2010). Hence, the expression of these proteins in differ-SH-SY5Y neural cells signifies the activation of neuronal features such as myelination and suppression of oncogenic characteristics of neuroblastoma cells.

The differ-SH-SY5Y neural cells also demonstrated a significant onefold downregulation of DDX39B and ribosomal proteins, RPS12, RPL10, RPL12 and RPSA. The DDX39B is a member of the DEAD-box family of RNA helicases that involve in pre-mRNA splicing and mRNA export to the cytoplasm (Zhang et al. 2018). Intrinsically, the DDX39B promotes the unwinding of the U4/U6 snRNA duplex, which in-turn permits the binding of the U2 snRNP to the premRNA in a series of ATP-dependent pre-mRNA splicing process (Shen et al. 2008). Consequently, DDX39B mediates the nuclear transport of mRNA by facilitating the interaction between THO complex and CIP29 and Aly (nuclear factors) (Folco et al. 2012). The interaction between the DDX39B with export proteins CIP29 and Aly during the formation of the conserved TREX mRNA export complex is controlled by ATP hydrolysis Dufu et al. (2010). A recent study has reported that the increased pre-ribosomal RNA levels of DDX39B augment global translation and cell proliferation of diverse cancer types. This study also showed that DDX39B knockdown cells displayed a significantly reduced stability of pre-ribosomal 47S RNA, whereas the 47S rRNA stability was unaltered in DDX39B overexpressed cells (Awasthi et al. 2018). The present study's findings suggest that the downregulation of DDX39B in differ-SH-SY5Y neural cells is closely linked to the suppression of ribosomal proteins and RNA biogenesis. The inhibition of cell proliferation in differentiated neural cells was caused by the suppression of ribosomal proteins as this event is pertinent in protein biosynthesis and cell growth. Evidence supporting this finding comes from a study by Linstrom et al. that suggested that silencing of ribosomal protein S9 (RPS9) elicits cell proliferation restriction mediated by the p53 tumour suppressor pathway in cancer cells. This study also pointed out that the suppression of ribosomal proteins effectively promotes differentiation processes, senescence, or apoptosis in rapidly propagating cancer cells (Lindström and Nistér 2010). According to our STRING PPI analysis, a total of 28 differentially regulated proteins were found to form the most prominent network in cluster II. Among these, 21 ribosomal proteins displayed a significant downregulation in differ-SHSY5Y neural cells compared to undifferentiated SH-SY5Y cells (Fig. 3A). These 21 ribosomal proteins were identified to have a pivotal role in the ribosome bio-pathway as curated by the KEGG database (Fig. 4).

The ribosome is the cellular translational machinery's principal component that decodes messenger RNA (mRNA) to produce an amino acid chain through a complementary anticodon sequence (Guimarães 2017). The manufacture of this machinery component is known as ribosome biogenesis and occurs in the nucleolus via initiation by the RNApolymerase-1 (RNA-pol1) that mediates the transcription of rRNA genes (Drygin et al. 2010). Three RNA polymerase enzymes, RNA-pol1, RNA-pol2, RNA-pol3 and nonribosomal factors, were responsible for generating the $90 \mathrm{~S}$ pre-ribosomes in the nucleolus undergoes several modifications before splitting into pre-60S and pre-40S particles (Torreira et al. 2017). These particles mature into the large (60S) and small (40S) subunits for protein synthesis during the transportation from nucleolus into cytoplasm compartments through dissociation from most of the non-ribosomal factors (Delavoie et al. 2019). Emerging studies have revealed that ribosome biogenesis and protein translations are exceptionally synchronized with cellular processes, including cell division, differentiation and growth (Zhou et al. 2015). Disruption in any of these two dynamic processes could impede the crucial cellular biological processes and well-being.

Furthermore, recent evidence has documented that downregulation of the ribosomal proteins (RPs) and translation efficiency during the differentiation process contribute to reduced cell growth (Marcon et al. 2017) (Bevort and Leffers 2000) (Hayashi et al. 2014). Bevort and Leffers have reported that 31 out of 32 RPs analysed were significantly suppressed in neuronal differentiation in human NTERA2 cells induced by retinoic acid. The same study also demonstrated that the reduction in RP mRNA expression was well correlated with inhibition of the proliferation marker known as proliferating cell nuclear antigen (PCNA) (Bevort and Leffers 2000). An incredible wealth of information was contributed by Hayashi and team elucidating the 
(A)

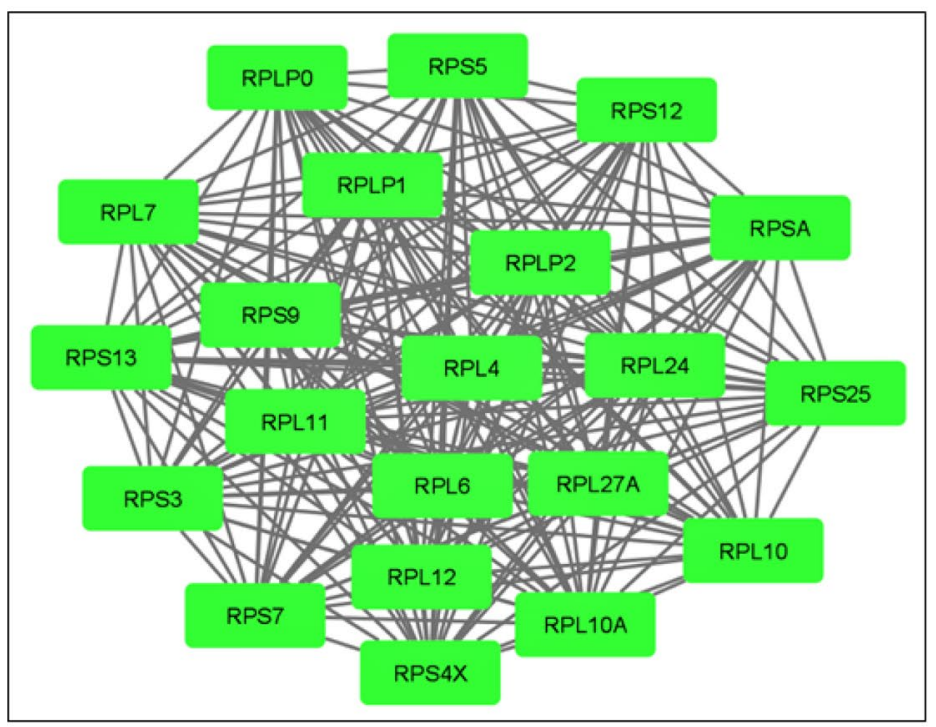

RIBOSOME

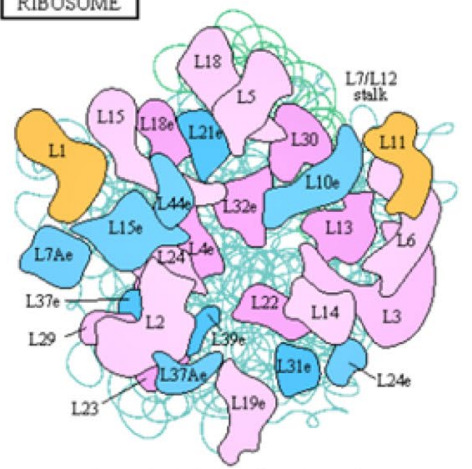

(B)

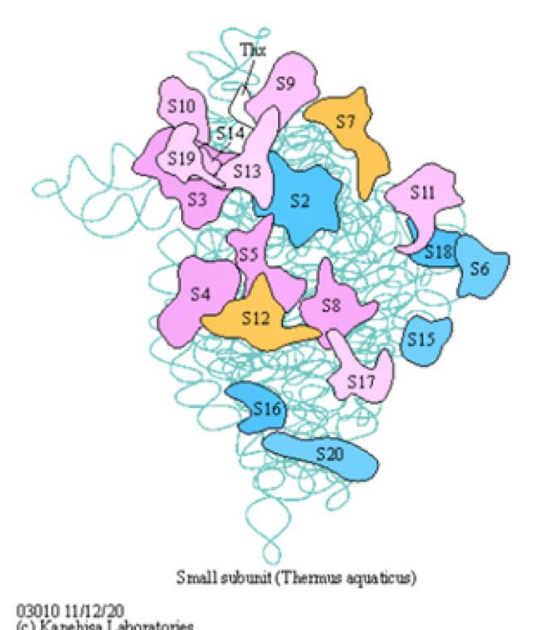

Rhosomal RNAs

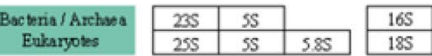

Rhosomal proteins
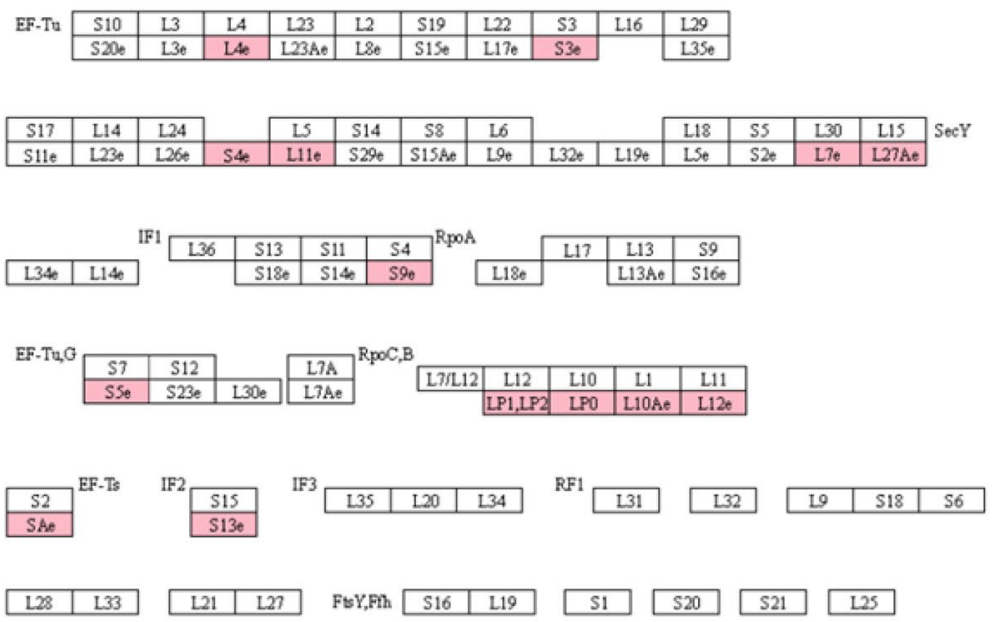

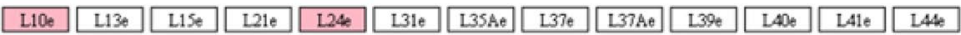

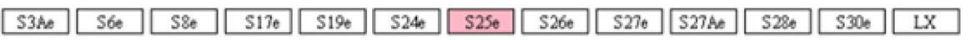

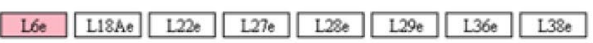

S7e $\$ 10 \% \quad S 128 \quad S 218$
Fig. 4 KEGG Pathway enrichment analysis of Cluster II proteins. (A) The differentially regulated cluster II protein network is visualized using Cytoscape 3.8.0. The green nodes indicate the significantly downregulated cluster II proteins $(p<0.05)$. (B) The position of cluster II proteins in the KEGG-ribosome pathway during the neuronal maturation in differ-SH-SY5Y neural cells are shown as pinkcoloured boxes. The cluster II proteins involved in this pathway are ribosomal protein family members that can be classified as large subunit ribosomal proteins (RPL10, RPL10A, RPL11, RPL12, RPL24, RPL27A, RPL4, RPL6, RPL7, RPLP0, RPLP1, RPLP2) and small subunit ribosomal proteins (RPS12, RPS13 RPS25, RPS3, RPS4X, RPS5, RPS7, RPS9, RPSA) ( Adapted from KEGG Ribosome pathway- ko03010) 
association between downregulation of rRNA transcription and cellular differentiation (Hayashi et al. 2014). According to this report, the rRNA transcription was deliberately downregulated using actinomycin D, a siRNA for Pol 1-specific transcription factor IA (TIF-IA) in HL-60 and THP-1 cells differentiation potential. The attenuation of rRNA transcription was shown to enhance the cell differentiation in both cell lines and increase the differentiation marker, CD11b. They also evaluated if cell differentiation was triggered by inhibition of the cell cycle since rRNA transcription is tightly paired with cell growth. The outcome of this study showed that cell cycle arrest that occurred without affecting rRNA transcription did not stimulate differentiation in mouse hematopoietic stem cells (Hayashi et al. 2014). This data is in line with our findings that showed a significant downregulation of RPs in differ-SH-SY5Y cells indicate the augmentation in the differentiation mechanism and suppression of cell growth compared to undifferentiated SH-SY5Y neuroblastoma cells.

The 6-OHDA induced neurodegeneration model is a wellacknowledged "simulation" conceived by the PD research community to study the cellular and molecular processes, both in vitro or in vivo settings (Xicoy et al. 2017). Although the model does not demonstrate the classical pathological hallmark of PD, which is the accumulation of $\alpha$-synuclein and Lewy bodies, it expresses the cardinal processes in $\mathrm{PD}$, including mitochondrial dysfunction, apoptosis, ROS induced oxidative stress, neuro-inflammation, lipid peroxidation and disruption in endogenous antioxidant enzymes (Dias et al. 2013). In our study, the 6-OHDA exposure on the differ-SH-SY5Y neural cells yielded a total of 101 differentially regulated proteins. It is important to highlight that none of these proteins exhibit close association to dopaminergic, adrenergic or cholinergic neuron receptors. The exposure of 6-OHDA on differ-SH-SY5Y neural cells revealed a remarkable onefold overexpression of VDAC1, HSPE1 and HSPA9 proteins (Fig. 1B).

The VDAC1 is found abundantly on the outer membrane of mitochondria and functions as a gatekeeper for the passage of ions $\left(\mathrm{Ca}^{2+}, \mathrm{K}^{+}, \mathrm{Na}^{+}\right)$and metabolite substrates ATP, ADP and Pi (Camara et al. 2017). The conformational states of VDAC1 are voltage-dependent and ion selectivity, exhibiting a preference to metabolite anions in high voltage conductance (open state) and cations in low voltage conductance (closed state) (Rostovtseva and Colombini 1997). The VDAC remains in high conductance or open state during mitochondria depolarization potential in the voltage range of about -40 to $+40 \mathrm{mV}$ (Hodge and Colombini 1997). The VDAC function is associated with NADH's oxidation, hence playing a pivotal role in mitochondria-mediated apoptotic signalling via interaction with pro-and anti-apoptotic mediators (Shoshan-Barmatz et al. 2017). Previous studies have reported that the overexpression of VDAC1 in cells undergoing apoptosis was mediated by increased cytosolic $\mathrm{Ca}^{2+}$ level. The treatment of pro-apoptosis inducer, hydrogen peroxide on Hela (human cervical adenocarcinoma) cells resulted in increased cytosolic $\mathrm{Ca}^{2+}$ and overexpressed oligomerised VDAC1 mediating the release of cytochrome c and apoptosis (Shoshan-Barmatz et al. 2017). Another study has further proven that a binding partner known as tubulin polymerization-promoting protein family member-3 (TPP3) promoted the oligomerisation of VDAC1 in palmitic acidinduced apoptosis of endothelial cells (Liu et al. 2020). Hence, the upregulation of VDAC1 protein expression in our finding indicates that the 6-OHDA treated differ-SH-SY5Y neural cells underwent rigorous oxidative stress-induced apoptosis, which is an expected outcome in a PD disease model.

The heat shock proteins (HSPs) are the most conserved $10 \mathrm{kDa}$ evolutionary proteins known as stress-inducible proteins. The HSPE1 (HSP10) is found abundantly in mitochondria, whereas HSPA9 (HSP70) exists in the cytoplasm and nucleus. Generally, HSPs function as cytoprotective proteins with related co-chaperones under oxidative stress induced apoptosis through the initiation of repair mechanism and refolding of misfolded peptides, possible proteolysis of irreparable proteins, signalling transduction and translocation (Sharma et al. 2012). Principally, the HSPA9 assists in the transportation of nuclear-encoded proteins to the mitochondria and these proteins are subsequently refolded by HSPD1(HSP60) and its co-chaperone, HSPE1 (Voos 2013). The HSPD1-HSPE1 complex chaperonin consists of two rings arranged to conform to a barrel-shaped structure with a central cavity. This complex receives the unfolded or misfolded proteins into its central cavity and facilitates the folding process divided into three steps. In the first step, the HSPD1-HSPE1 complex binds firmly with the unfolded or misfolded proteins; then the proteins get trapped in the central cavity capped by HSPE1 refolding of the protein takes place. Finally, the correctly folded proteins are ejected from the chaperonin complex. The unfolded or partially folded proteins are redirected to the chaperonin to repeat the entire cycle until the correctly folded proteins are achieved (Jia et al. 2011). While the HSPD1 and HSPE1 are crucial housekeeping proteins for efficient mitochondria function and biogenesis, the HSPA9 is best known for its cytosolic chaperone activity in assisting protein folding, degradation and translocation. Overwhelming evidence has pointed out that HSPs have been identified to be involved in multiple pathways in promoting the anti-apoptosis effect (Ikwegbue et al. 2018). According to Li et al. the chaperone activity of HSPA9 is critical for the inhibition of caspase activation at a reaction point between the cytochrome c release and caspase-3 activation ( $\mathrm{Li}$ et al. 2000). The overexpression of HSPA9 was also reported to indirectly inhibit the stress-induced apoptosis by preventing the conformational 
change and translocation of Bax into mitochondria, indicating the suppression of apoptosis through Bax inactivation and inhibition of caspase activation (Stankiewicz et al. 2005). A recent study has clarified that overexpression of HSPA9 prevents inflammation in a rat model of intracerebral haemorrhage through inhibition of inflammatory cytokines such as TNF- $\alpha$, IL- $\beta$, Bax, and increased Bcl-2 levels (Lv et al. 2017). Hence, we postulate that the overexpression of HSPE1 and HSPA9 in our study is closely connected to the cytoprotective mechanism of the neuronal cells to inhibit the avalanche of apoptotic mediators released in response to 6-OHDA treatment. The HSP expression in the PD cell

(A)
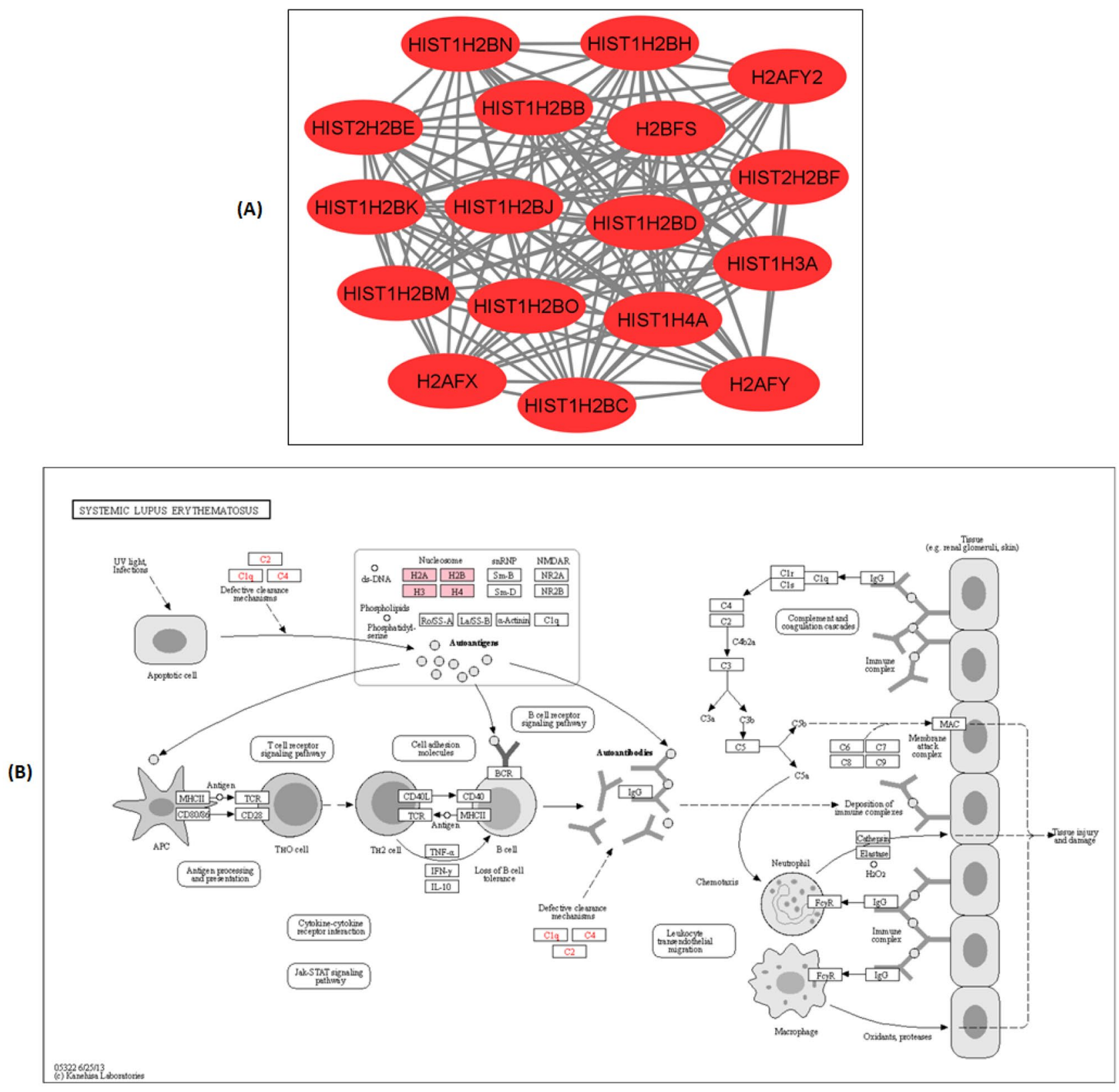

Fig. 5 KEGG pathway enrichment analysis of cluster III proteins. (A) The differentially regulated cluster III protein network in differSH-SY5Y neural cells in response to 6-OHDA is visualized using Cytoscape 3.8.0. The Red nodes indicate the significantly upregulated cluster III proteins $(p<0.05)$. (B) The position of cluster III proteins in the KEGG SLE pathway during the 6-OHDA induced neurodegeneration process are shown as pink-coloured boxes. The 17 cluster III proteins involved in this pathway are from H2A histone family mem- ber (H2AFX, H2AFY, H2AFY2), Histone H2B type (H2BFS), Histone cluster 1 H2B family (HIST1H2BB, HIST1H2BC, HIST1H2BD, HIST1H2BH, HIST1H2BJ, HIST1H2BK, HIST1H2BM, HIST1H2BN, HIST1H2BO), Histone cluster $1 \mathrm{H} 3$ family (HIST1H3A), Histone cluster $1 \mathrm{H} 4$ family (HIST1H4A) and Histone cluster $2 \mathrm{H} 2 \mathrm{~B}$ family (HIST2H2BE, HIST2H2BF) ( Adapted from KEGG Systemic Lupus Erythematosus pathway- hsa05322) 
model is an important biomarker that paves the way for future studies in developing potential therapies targeting the HSPE1 and HSPA9 to mitigate apoptosis-induced cell death.

The treatment of 6-OHDA-induced neurodegeneration on differ-SH-SY5Y neural cells resulted in an enrichment of 4 clusters or pathways in STRING PPI analysis (Fig. 3B). The crosstalk between proteins populated in cluster IV of STRING PPI displaying the most prominent interaction was further investigated using KEGG pathway enrichment analysis. The KEGG bioinformatic pathway enrichment database predicted the systemic lupus erythematosus (SLE) pathway with the involvement of 17 out of 19 upregulated proteins (Fig. 5). SLE is an autoimmune disease of unknown aetiology that primarily affects women in the childbearing age (Pieterse and van der Vlag 2014). The disease is characterized by disturbances of the immune system that arise when the immune cells respond to self-antigens, mainly nuclear constituents, i.e. histones, ribonucleoproteins and DNA (Pradhan et al. 2010). For over two decades, apoptosis has been regarded as a significant source of autoantigens in SLE. Apoptosis can be actively triggered by ligation of cell surface receptors, including Fas and tumour necrosis factor receptor (TNFR) or passively via deficient in crucial cell survival signals (De Wilde et al. 2001). Apoptotic cells undergo an orderly process of morphological alterations, such as nuclear chromatin condensation, nuclear splitting, cytoskeletal disruption, cell shrinkage and membrane blebbing (Saraste and Pulkki 2000). Persistent exposure of the immune system to apoptotic bodies leads to the formation of an anti-chromatin/chromatin complex, triggering an array of inflammation in multiple organs in SLE patients. Previous studies have shown that autoantibodies' increased reactivity against histone proteins, namely histone $\mathrm{H} 4$ and H2B peptides, correlated with SLE disease activity (Dieker et al. 2016). Whereas in PD, the selective vulnerability of dopamine neurons to environmental toxins, intracellular accumulations of highly oxidative free radical and toxic accumulation of misfolded proteins leads to neurodegeneration (apoptosis) and activation of neuro-inflammation pathway (Hald and Lotharius 2005). As such, the neuroinflammation pathway is triggered through activation of microglia or the "immune cell of the brain", which releases the pro-inflammatory mediators including tumour necrosis factor (TNF- $\alpha$ ), interleukin (IL-1 $\beta$ ), IL-2, IL-4, interferon (IFN- $\gamma$ ), and nitric oxide (NO) (Liu et al. 2019). The proinflammatory mediators instigate further detrimental effects on nigral neurons by causing direct toxicity in neurons and induce ongoing neuro-inflammation through microglial NO activation (Jung et al. 2019). Emerging studies have reported that chronic SLE patients presented with parkinsonian syndromes, including slowness in movement, adiadochokinesia, postural rigidity, and tremor (Fabiani et al. 2002).
In our study, the histone proteins were shown to be exceptionally overexpressed in 6-OHDA induced neurodegeneration on differ-SH-SY5Y neural cells (PD cell model). Structurally, histone proteins exist as an octamer with two copies of each of the four core histone proteins $\mathrm{H} 2 \mathrm{~A}, \mathrm{H} 2 \mathrm{~B}, \mathrm{H} 3$, and $\mathrm{H} 4$ and two linker histones, $\mathrm{H} 1$ and $\mathrm{H} 5$ (Andrews and Luger 2011). The 147 bp of DNA packages the octamer histones to form a nucleosome. The four core histones form a conserved central motif domain known as histone fold composed of a long central $\alpha$-helix structure with a short helix flanked on either side (Jiang and Pugh 2009). In comparison, the linker histone associates with nucleosome to transform nucleosomes into various high-order chromatin structures. Ideally, the histone proteins function in constructing the nucleus and fine-tuning gene expression for physiological and pathological processes (Martire and Banaszynski 2020). An increasing body of evidence suggests that besides regulating nucleosome dynamics, histone proteins are released in the extracellular field by cells undergoing apoptosis (Silk et al. 2017).

It is well established that chromatin condensation coupled with DNA fragmentation is important nuclear events during apoptosis (He et al. 2009). In response to apoptotic signals, the core and linker histones are detached from genomic DNA and released into cytoplasmic and extracellular regions. Wu et al. have reported that the timing of histone release from genomic DNA directly correlated with the advancement of the apoptosis process (Wu et al. 2002). The release of histone proteins and DNA-bound nucleosomes into the intracellular space by damaged DNA is an essential indicator of apoptosis-activated pro-inflammatory cascades (Silk et al. 2017). Once in the cytosol, histones act as a member of the damage-associated molecular pattern molecules (DAMPs), activating immune response and causing additional cytoproliferative effects (Xu et al. 2009). Along these lines, studies have revealed that core histones such as $\mathrm{H} 2 \mathrm{~A}, \mathrm{H} 2 \mathrm{~B}, \mathrm{H} 3, \mathrm{H} 4$ and linker histone $\mathrm{H} 1$ are frequently detected in neurons (Mishra et al. 2010), microglia (Klein et al. 2014) and macrophages (Brix et al. 1998) in response to oxidative stress (Hu et al. 2018). Notably, the degree of circulating histone and nucleosomes are elevated in cancer, infection and inflammation proposing histone as an essential biomarker in human diseases (Chen et al. 2014). Hence, the upregulation of a set of histone proteins in 6-OHDA induced neuronal death in our study is a clear indication of chromatin condensation and modification during DNA fragmentation in the apoptosis process.

In conclusion, the PD cell model is an essential in vitro platform for investigating the structural and molecular changes manifested in disease process and paving the way for future discoveries of potential therapeutic drugs that reverse these mechanisms. Ideally, a disease model should express the biomarkers that indicate the progression of PD's 
pathological conditions such as mitochondrial dysfunction, DNA fragmentation, neurodegeneration, and neuroinflammation. The differ-SH-SY5Y neural cells developed from SH-SY5Y human neuroblastoma cells expressed substantial dopaminergic markers and, upon perturbation with 6-OHDA, displayed pathological changes frequently detected in PD. The differentially regulated proteins were analysed using GO functional annotation, STRING PPI and KEGG pathway enrichment databases to understand the cellular machinery's alterations during the disease process. We suggest that the downregulation of the ribosome pathway mediates the differentiation of SH-SY5Y neuroblastoma cells and nucleosomal degradation demonstrated by upregulation of histone products in SLE pathway is a key event in 6-OHDA induced neurodegenerative process.

Supplementary Information The online version contains supplementary material available at https://doi.org/10.1007/s12031-021-01962-z.

Acknowledgements The authors thank Dr Syafiq Asnawi (Proteomic laboratory Jeffrey Cheah School of Medicine, Monash University Malaysia) for technical support in carrying out the LC-MS/MS analysis.

Author Contribution KBM performed all experiments and prepared the manuscript, AR and HN designed the overall study. AR and SDS involved in project administration. PR assisted in data analysis. All authors read and approved the final manuscript.

Funding Open Access funding enabled and organized by CAUL and its Member Institutions. This study was supported by the International Medical University, Kuala Lumpur research grant (Grant: IMU R 194 2016).

\section{Declarations}

Ethics Approval and Consent to Participate Not applicable.

Consent for Publication Permission obtained from Kanehisa Laboratories to publish the KEGG pathway map images both in prints and digital under the CC BY 4.0 open access license.

Competing Interests The authors declare no competing interests.

Open Access This article is licensed under a Creative Commons Attribution 4.0 International License, which permits use, sharing, adaptation, distribution and reproduction in any medium or format, as long as you give appropriate credit to the original author(s) and the source, provide a link to the Creative Commons licence, and indicate if changes were made. The images or other third party material in this article are included in the article's Creative Commons licence, unless indicated otherwise in a credit line to the material. If material is not included in the article's Creative Commons licence and your intended use is not permitted by statutory regulation or exceeds the permitted use, you will need to obtain permission directly from the copyright holder. To view a copy of this licence, visit http://creativecommons.org/licenses/by/4.0/.

\section{References}

Andrews AJ, Luger K (2011) Nucleosome structure(s) and stability: Variations on a theme. Annu Rev Biophys 40:99-117

Arsikin K, Kravic-Stevovic T, Jovanovic M, Ristic B, Tovilovic G, Zogovic N, Bumbasirevic V, Trajkovic V, Harhaji-Trajkovic L (2012) Autophagy-dependent and -independent involvement of AMP-activated protein kinase in 6-hydroxydopamine toxicity to SH-SY5Y neuroblastoma cells. Biochimi Biophys Acta Mol Basis Dis 1822:1826-1836

Aslam B, Basit M, Nisar MA, Khurshid M, Rasool MH (2017) Proteomics: Technologies and their applications. J Chromatogr Sci 55:182-196

Awasthi S, Chakrapani B, Mahesh A, Chavali PL, Chavali S, Dhayalan A (2018) DDX39B promotes translation through the regulation of pre-ribosomal RNA levels. RNA Biol 15:1157-1166

Bevort M, Leffers H (2000) Down regulation of ribosomal protein mRNAs during neuronal differentiation of human NTERA2 cells. Differentiation 66:81-92

Brix K, Summa W, Lottspeich F, Herzog V (1998) Extracellularly occurring histone $\mathrm{H} 1$ mediates the binding of thyroglobulin to the cell surface of mouse macrophages. J Clin Invest 102:283-293

Camara A, Zhou Y, Wen PC, Tajkhorshid E, Kwok WM (2017) Mitochondrial VDAC1: A key gatekeeper as potential therapeutic target. Front Physiol 8:460

Chen R, Kang R, Fan XG, Tang D (2014) Release and activity of histone in diseases. Cell Death Dis 5:e1370

Cheung Y-T, Lau WK-W, Yu M-S, Lai CS-W, Yeung S-C, So K-F, Chang RC-C (2009) Effects of all-trans-retinoic acid on human SH-SY5Y neuroblastoma as in vitro model in neurotoxicity research. Neurotoxicology 30:127-135

De Wilde G, Murray-Rust J, Boone E, Olerenshaw D, McDonald NQ, Ibanez C, Haegeman G, Wollmer A, Federwisch M (2001) Structure-activity relationship of the p55 TNF receptor death domain and its lymphoproliferation mutants. Eur J Biochem 268:1382-1391

Delavoie F, Soldan V, Rinaldi D et al (2019) The path of pre-ribosomes through the nuclear pore complex revealed by electron tomography. Nat Commun 10:1-12

Dias V, Junn E, Mouradian MM (2013) The role of oxidative stress in parkinson's disease. J Parkinsons Dis 3:461-491

Dieker J, Berden JH, Bakker M, Briand JP, Muller S, Voll R, Sjöwall C, Herrmann M, Hilbrands LB, van der Vlag J (2016) Autoantibodies against modified histone peptides in SLE patients are associated with disease activity and lupus nephritis. PLoS One 11:e0165373

Drygin D, Rice WG, Grummt I (2010) The RNA polymerase i transcription machinery: An emerging target for the treatment of cancer. Annu Rev Pharmacol Toxicol 50:131-156

Dudek E, Michalak M (2013) Calnexin and Calreticulin. In: Encyclopedia of Metalloproteins. Springer New York

Dufu K, Livingstone MJ, Seebacher J, Gygi SP, Wilson SA, Reed $\mathrm{R}$ (2010) ATP is required for interactions between UAP56 and two conserved mRNA export proteins, Aly and CIP29, to assemble the TREX complex. Genes Dev 24:2043-2053

El-Rami F, Nelson K, Xu P (2017) Proteomic approach for extracting cytoplasmic proteins from Streptococcus sanguinis using mass spectrometry. J Mol Biol Res 7:50

Fabiani G, Teive HA, Germiniani FM, Sá DS, Werneck LC (2002) Reversible parkinsonian syndrome in systemic and brain vasculitis. Mov Disord: Official Journal of the Movement Disorder Society 17:601-604 
Filograna R, Civiero L, Ferrari V, Codolo G, Greggio E, Bubacco L, Beltramini M, Bisaglia M, Castresana JS (2015) Analysis of the catecholaminergic phenotype in human SH-SY5Y and BE(2)M17 neuroblastoma cell lines upon differentiation. PLoS One 10:e0136769

Folco EG, Lee CS, Dufu K, Yamazaki T, Reed R (2012) The proteins PDIP3 and ZC11A associate with the human TREX complex in an ATP-dependent manner and function in mRNA export. PLoS One 7:e43804

Gomez-Lazaro M, Bonekamp NA, Galindo MF, Jordán J, Schrader M (2008) 6-Hydroxydopamine (6-OHDA) induces Drp1-dependent mitochondrial fragmentation in SH-SY5Y cells. Free Radic Biol Med 44:1960-1969

Guimarães RC (2017) Self-referential encoding on modules of anticodon pairs-Roots of the biological flow system. Life (Basel, Switzerland) 7:16

Hald A, Lotharius J (2005) Oxidative stress and inflammation in Parkinson's disease: Is there a causal link? Exp Neurol 193:279-290

Hashemi SH, Li JY, Ahlman H, Dahlström A (2003) SSR2(a) Receptor expression and adrenergic/cholinergic characteristics in differentiated SH-SY5Y cells. Neurochem Res 28:449-460

Hayashi Y, Kuroda T, Kishimoto H, Wang C, Iwama A, Kimura K (2014) Downregulation of rRNA transcription triggers cell differentiation. PLoS One 9:e98586

He B, Lu N, Zhou Z (2009) Cellular and nuclear degradation during apoptosis. Curr Opin Cell Biol 21:900-912

Hodge T, Colombini M (1997) Regulation of metabolite flux through voltage-gating of VDAC channels. J Membr Biol 157:271-279

Hu J, Han J, Li H, Zhang X, Liu LL, Chen F, Zeng B (2018) Human embryonic kidney 293 cells: A vehicle for biopharmaceutical manufacturing, structural biology, and electrophysiology. Cells Tissues Organs 205:1-8

Iglesias-González J, Sánchez-Iglesias S, Méndez-Álvarez E, Rose S, Hikima A, Jenner P, Soto-Otero R (2012) Differential toxicity of 6-hydroxydopamine in SH-SY5Y human neuroblastoma cells and rat brain mitochondria: Protective role of catalase and superoxide dismutase. Neurochem Res 37:2150-2160

Ikwegbue PC, Masamba P, Oyinloye BE, Kappo AP (2017) Roles of heat shock proteins in apoptosis, oxidative stress, human inflammatory diseases, and cancer. Pharmaceuticals (Basel, Switzerland) 11:2

Jia H, Halilou AI, Hu L, Cai W, Liu J, Huang B (2011) Heat shock protein 10 (Hsp10) in immune-related diseases: one coin, two sides. Int J Biochem Mol Biol 2:47-57

Jiang C, Pugh BF (2009) Nucleosome positioning and gene regulation: Advances through genomics. Nat Rev Genet 10:161-172

Jung YJ, Tweedie D, Scerba MT, Greig NH (2019) Neuroinflammation as a factor of neurodegenerative disease: Thalidomide analogs as treatments. Front Cell Dev Biol 7:313

Kanehisa M (2019) Toward understanding the origin and evolution of cellular organisms. Protein Sci 28:1947-1951

Katsuyama M, Kimura E, Ibi M, Iwata K, Matsumoto M, Asaoka N, Yabe-Nishimura C (2021) Clioquinol inhibits dopamine- $\beta$ hydroxylase secretion and noradrenaline synthesis by affecting the redox status of ATOX1 and copper transport in human neuroblastoma SH-SY5Y cells. Arch Toxicol 95:135-148

Khwanraj K, Phruksaniyom C, Madlah S, Dharmasaroja P (2015) Neurol Res Int 2015:734703

Klein B, Lütz-Meindl U, Kerschbaum HH (2014) From the nucleus to the plasma membrane: Translocation of the nuclear proteins histone $\mathrm{H} 3$ and lamin B1 in apoptotic microglia. Apoptosis 19:759-775

Kovalevich J, Langford D (2013) Considerations for the use of SHSY5Y neuroblastoma cells in neurobiology. Methods Mol Biol 1078:9-21

Kraus A, Groenendyk J, Bedard K, Baldwin TA, Krause KH, DuboisDauphin M, Dyck J, Rosenbaum EE, Korngut L, Colley, et al
(2010) Calnexin deficiency leads to dysmyelination. J Biol Chem 285:18928-18938

Lee AC, Shih YY, Zhou F, Chao TC, Lee H, Liao YF, Hsu WM, Hong $\mathrm{JH}$ (2019) Calreticulin regulates MYCN expression to control neuronal differentiation and stemness of neuroblastoma. J Mol Med (Berlin, Germany) 97:325-339

Li CY, Lee JS, Ko YG, Kim JI, Seo JS (2000) Heat shock protein 70 inhibits apoptosis downstream of cytochrome c release and upstream of caspase-3 activation. J Biol Chem 275:25665-25671

Lindström MS, Nistér M (2010) Silencing of ribosomal protein S9 elicits a multitude of cellular responses inhibiting the growth of cancer cells subsequent to p53 activation. PLoS One 5:e9578

Liu CY, Wang X, Liu C, Zhang HL (2019) Pharmacological targeting of microglial activation: New therapeutic approach. Front Cell Neurosci 13:514

Liu N, Li Y, Nan W, Zhou W, Huang J, Li R, Zhou L, Hu R (2020) Interaction of TPPP3 with VDAC1 promotes endothelial injury through activation of reactive oxygen species. Oxid Med Cell Longev 2020:5950195

Lopes FM, da Motta LL, De Bastiani MA, Pfaffenseller B, Aguiar BW, de Souza LF, Zanatta G, Vargas DM, Schönhofen P, Londero GF et al (2017) RA differentiation enhances dopaminergic features, changes redox parameters, and increases dopamine transporter dependency in 6-hydroxydopamine-induced neurotoxicity in SHSY5Y cells. Neurotox Res 31:545-559

Lv LJ, Li J, Qiao HB, Nie BJ, Lu P, Xue F, Zhang ZM (2017) Overexpression of GRP75 inhibits inflammation in a rat model of intracerebral hemorrhage. Mol Med Rep 15:1368-1372

Magalingam KB, Radhakrishnan AK, Somanath SD, Md S, Haleagrahara N (2020) Influence of serum concentration in retinoic acid and phorbol ester induced differentiation of SH-SY5Y human neuroblastoma cell line. Mol Biol Rep 47:8775-8788

Marcon BH, Holetz FB, Eastman G, Origa-Alves AC, Amorós MA, de Aguiar AM, Rebelatto CK, Brofman P, Sotelo-Silveira J, Dallagiovanna B (2017) Downregulation of the protein synthesis machinery is a major regulatory event during early adipogenic differentiation of human adipose-derived stromal cells. Stem Cell Res 25:191-201

Marei H, El-Gamal A, Althani A, Afifi N, Abd-Elmaksoud A, Farag A, Cenciarelli C, Thomas C, Anwarul H (2018) Cholinergic and dopaminergic neuronal differentiation of human adipose tissue derived mesenchymal stem cells. J Cell Physiol 233:936-945

Martire S, Banaszynski LA (2020) The roles of histone variants in fine-tuning chromatin organization and function. Nat Rev Mol Cell Biol 21:522-541

Mi H, Muruganujan A, Ebert D, Huang X, Thomas PD (2019) PANTHER version 14: More genomes a new PANTHER GO-slim and improvements in enrichment analysis tools. Nucleic Acids Res 47:D419-D426

Mishra B, von der Ohe M, Schulze C, Bian S, Makhina T, Loers G, Kleene R, Schachner M (2010) Functional role of the interaction between polysialic acid and extracellular histone H1. J Neurosci 30:12400-12413

Pieterse E, van der Vlag J (2014) Breaking immunological tolerance in systemic lupus erythematosus. Front Immunol 5:164

Pomaznoy M, Ha B, Peters B (2018) GOnet: A tool for interactive Gene Ontology analysis. BMC Bioinform 19:470

Pradhan VD, Patwardhan MM, Ghosh K (2010) Anti-nucleosome antibodies as a disease marker in systemic lupus erythematosus and its correlation with disease activity and other autoantibodies. Indian J Dermatol Venereol Leprol 76:145-149

Rostovtseva T, Colombini M (1997) Vdac channels mediate and gate the flow of ATP: Implications for the regulation of mitochondrial function. Biophys J 72:1954-1962

Saraste A, Pulkki K (2000) Morphologic and biochemical hallmarks of apoptosis. Cardiovasc Res 45:528-537 
Sharma GT, Nath A, Prasad S, Singhal S, Singh N, Gade NE, Dubey PK, Saikumar G (2012) Expression and characterization of constitutive heat shock protein 70.1 (HSPA-1A) gene in in vitro produced and in vivo-derived buffalo (Bubalus bubalis) embryos. Reprod Domest Anim 47:975-983

Shen H, Zheng X, Shen J, Zhang L, Zhao R, Green MR (2008) Distinct activities of the DExD/H-box splicing factor hUAP56 facilitate stepwise assembly of the spliceosome. Genes Dev 22:1796-1803

Shoshan-Barmatz V, Maldonado EN, Krelin Y (2017) VDAC1 at the crossroads of cell metabolism, apoptosis and cell stress. Cell Stress 1:11-36

Silk E, Zhao H, Weng H, Ma D (2017) The role of extracellular histone in organ injury. Cell Death Dis 8:e2812

Sinitcyn P, Rudolph JD, Cox J (2018) Computational methods for understanding mass spectrometry-based shotgun proteomics data. Annu Rev Biomed Data Sci 1:207-234

Stankiewicz AR, Lachapelle G, Foo CP, Radicioni SM, Mosser DD (2005) Hsp70 inhibits heat-induced apoptosis upstream of mitochondria by preventing Bax translocation. J Biol Chem 280:38729-38739

Storch A, Kaftan A, Burkhardt K, Schwarz J (2000) 6Hydroxydopamine toxicity towards human SH-SY5Y dopaminergic neuroblastoma cells: independent of mitochondrial energy metabolism. J Neural Transm 107:0281-0293

Szklarczyk D, Gable AL, Lyon D, Junge A, Wyder S, Huerta-Cepas J, Simonovic M, Doncheva NT, Morris JH, Bork P, Jensen LJ, Mering CV (2019) STRING v11: Protein-protein association networks with increased coverage supporting functional discovery in genome-wide experimental datasets. Nucleic Acids Res 47:D607-D613

Torreira E, Louro JA, Pazos I, González-Polo N, Gil-Carton D, Duran AG, Tosi S, Gallego O, Calvo O, Fernández-Tornero C (2017)
The dynamic assembly of distinct RNA polymerase i complexes modulates rDNA transcription. Elife 6:e20832

Voos W (2013) Chaperone-protease networks in mitochondrial protein homeostasis. Biochim Biophys Acta - Mol Cell Res 1833:388-399

Wu D, Ingram A, Lahti JH, Mazza B, Grenet J, Kapoor A, Liu L, Kidd VJ, Tang D (2002) Apoptotic release of histones from nucleosomes. J Biol Chem 277:12001-12008

Xiao X, Chen C, Yu TM, Ou J, Rui M, Zhai Y, He Y, Xue L, Ho MS (2017) Molecular chaperone calnexin regulates the function of Drosophila sodium channel paralytic. Front Mol Neurosci 10:57

Xicoy H, Brouwers JF, Kalnytska O, Wieringa B, Martens GJM (2020) Lipid analysis of the 6-hydroxydopamine-treated SH-SY5Y cell model for Parkinson's disease. Mol Neurobiol 57:848-859

Xicoy H, Wieringa B, Martens GJM (2017) The SH-SY5Y cell line in Parkinson's disease research: a systematic review. Mol Neurodegener 12:1-11

Xu J, Zhang X, Pelayo R, Monestier M, Ammollo CT, Semeraro F, Taylor FB, Esmon NL, Lupu F, Esmon CT (2009) Extracellular histones are major mediators of death in sepsis. Nat Med 15:1318-1321

Zhang L, Yang Y, Li B, Scott IC, Lou X (2018) The DEAD-box RNA helicase Ddx39ab is essential for myocyte and lens development in zebrafish. Development (Cambridge, England) 14:dev161018

Zhou X, Liao WJ, Liao JM, Liao P, Lu H (2015) Ribosomal proteins: functions beyond the ribosome. J Mol Cell Biol 7:92-104

Publisher's Note Springer Nature remains neutral with regard to jurisdictional claims in published maps and institutional affiliations. 